Stölzle, W./ Hofmann, E./ Hofer, F. (2005):

Supply Chain Costing: Konzeptionelle Grundlagen und ausgewählte Instrumente.

In: Brecht, U. (Hrsg.): Neue Entwicklungen im Rechnungswesen: Prozesse optimieren, Berichtswesen anpassen, Kosten senken. Wiesbaden 2005, S. 51-85.

Prof. Dr. Wolfgang Stölzle, Dr. Erik Hofmann \& Florian Hofer Kühne-Institut für Logistik (KLOG-HSG)

Universität St.Gallen

Dufourstrasse 40a

CH-9000 St.Gallen 


\section{Supply Chain Costing: Konzeptionelle Grundlagen und ausgewählte Instrumente}

Prof. Dr. Wolfgang Stölzle, Vorsitzender Direktor des Kühne-Instituts für Logistik an der Universität St.Gallen, Schweiz

Dr. Erik Hofmann, Projektleiter und Habilitand am Kühne-Institut für Logistik an der Universität St.Gallen, Schweiz

Dipl.-Wirtsch.-Ing. Florian Hofer, Wissenschaftlicher Mitarbeiter am Kühne-Institut für Logistik der Universität St.Gallen, Schweiz

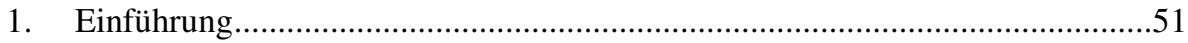

2. Konzeptionelle Grundlagen des Supply Chain Costing .................................52

2.1. Von der Logistik zum Supply Chain Management..................................53

2.2. Von der Kostenrechnung zum Kostenmanagement....................................57

2.3. Logistikkostenrechnung als Referenzpunkt des Kostenmanagement in Supply Chains............................................................................60

2.4. Anforderungen an das Kostenmanagement in Supply Chains ...................64

3. Ausgewählte Instrumente für das Supply Chain Costing ...............................69

3.1. Lebenszyklusorientierte Kostenrechnung...............................................69

3.2. Unternehmensübergreifende Prozesskostenrechnung ...............................72

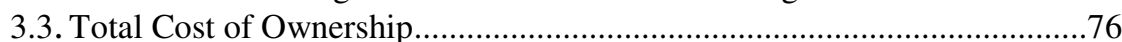

4. Zusammenfassung und Ausblick.................................................................... 78 


\section{Einführung}

Die Zusammenarbeit von Unternehmen in Wertschöpfungsnetzwerken nimmt zur Realisierung der strategischen Unternehmensziele eine immer bedeutendere Stellung ein. Zentrale Aspekte sind neben der Globalisierung wirtschaftlichen Handelns steigende Kundenanforderungen an Produkte und Dienstleistungen sowie die Ausrichtung des unternehmerischen Handelns an den Interessen der Anteilseigner. Damit einher gehen ein erhöhter Wettbewerbs- und Kostendruck, der sich nicht nur auf einzelne Unternehmen, sondern auch auf die Akteure und Prozesse in Wertschöpfungsnetzwerken auswirkt. Mit Hilfe des Supply Chain Management, bei dem sowohl das unternehmensübergreifende Management von Material-, Waren-, Informations- und Finanzflüssen entlang der Wertschöpfungsstufen als auch das Management von Kooperationen im Fokus der Betrachtung stehen, wird diesen Anforderungen Rechnung getragen. Die Ziele des Supply Chain Management bestehen u.a. darin, unternehmensübergreifende Transparenz von Leistungen (Bedarfe, Bestände und Kapazitäten) und Kosten zu erreichen. Ferner sollen sich den Supply Chain Management-Überlegungen folgend Entscheidungsträger auf verschiedenen Wertschöpfungsstufen nicht nur von Unternehmensinteressen leiten lassen, sondern nach einem unternehmensübergreifenden Gesamtoptimum suchen. ${ }^{1}$

Der Anspruch, von einer unternehmensbezogenen auf eine unternehmensübergreifende Betrachtungsweise überzugehen, unterstreicht die Bedeutung organisatorischer Fragestellungen im Supply Chain Management. ${ }^{2}$ Diesbezüglich findet sich vermehrt die Forderung, Informationen über die Kostensituation der berücksichtigten Supply ChainAkteure verursachungsgerecht zu ermitteln und den relevanten Entscheidungsträgern auf den einzelnen Wertschöpfungsstufen zeitgerecht bereit zu stellen. Die unternehmensübergreifende Betrachtung legt es nahe, argumentativ am Supply Chain Management anzuknüpfen und aus dieser Perspektive zu beurteilen, wie das Kostenmanagement zu integrieren ist. Mit der Entwicklung des Kostenmanagement als Gesamtheit aller Steuerungsmaßnahmen ist die zielorientierte Beeinflussung des Niveaus sowie der Strukturen und Verläufe von Kosten verbunden. ${ }^{3}$ Während sich in der Literatur die Instrumente des Kostenmanagement in der Regel auf einzelne Unternehmen und die dort anfallenden Kosten beziehen (intraorganisatorische Kostenbetrachtung), sollte beim Kostenmanagement in Supply Chains eine betont unternehmensübergreifende, wertschöpfungskettenumfassende Analyse und Gestaltung der dort anfallenden Kosten im Vordergrund stehen (interorganisatorische Kostenbetrachtung). Diese Forderung zeigt die Notwendigkeit nach einer Weiterentwicklung des Kostenmanagement auf, um Entscheidungen im Kontext von Wertschöpfungsnetzen zu fundieren. ${ }^{4}$ Mit der Integration des unternehmensübergreifenden Kostenmanagement erfährt das Konzept des Supply Chain Management

Vgl. Bechtel/Jayaram (1997), S. 21; Cooper/Ellram (1993), S. 13f.

Vgl. Stölzle (2002b), S. 283.

Vgl. Franz/Kajüter (2002), S. 8.

Vgl. Seuring (2001), S. 1. 
über die Überlegungen des internen Rechnungswesens eine systematische Erweiterung. Es besteht somit Bedarf an einem unternehmensübergreifenden Kosten-- und Erlösmanagement, das in Wertschöpfungsnetzwerken terminologisch korrekt als "Demand \& Supply Net Cost Management" bezeichnet werden müsste, in der betriebswirtschaftlichen Literatur aber "Supply Chain Costing" genannt wird. ${ }^{1}$

Der vorliegende Beitrag widmet sich den konzeptionellen Grundlagen und Instrumenten des Supply Chain Costing. Basierend auf der Entwicklung von der Logistik zum Supply Chain Management sowie der Kostenrechung zum Kostenmanagement werden in Abschnitt 2 die konzeptionellen Anforderungen dargestellt, die in die Überlegungen des Kostenmanagement in Supply Chains Eingang finden. Aufgrund ihres Querschnittscharakters kann die Logistikkostenrechnung als Referenzpunkt herangezogen werden. Auch wenn die betriebswirtschaftliche Forschung dem Ansatz des Supply Chain Costing durchaus Handlungsbedarf einräumt, stehen die wissenschaftlichen Ausführungen bisweilen am Anfang und lassen viel Raum für vertiefende Untersuchungen. Deshalb werden in Abschnitt 3 Methoden und Instrumente zur Gestaltung und Analyse von unternehmensübergreifenden Kosten zur Realisierung des Supply Chain Costing vorgestellt. Der Aufsatz schließt in Abschnitt 4 mit einem Ausblick auf weiterführende Anstrengungen in Wissenschaft und Praxis.

\section{Konzeptionelle Grundlagen des Supply Chain Costing}

Eine Annäherung an das Supply Chain Costing soll durch eine historische Betrachtung der konstitutionellen Elemente des Supply Chain Management einerseits und des Kostenmanagement andererseits erfolgen. Ebenso gehen von der Logistikkostenrechnung Impulse für das Supply Chain Costing aus. Damit werden die Entwicklungszüge "von der Logistik zum Supply Chain Management" und "von der Kostenrechnung zum Kostenmanagement" aufgegriffen, um systematisch den Bedarf nach einem Supply Chain Costing abzuleiten.

Mit der unternehmensübergreifenden Koordination und Integration von Unternehmensprozessen kommt dem aus der betriebswirtschaftlichen Logistikkonzeption erwachsenen Konzept des Supply Chain Management eine bedeutende Rolle zu. Die Planung, Realisierung und Steuerung der unternehmensübergreifenden Prozesse erfolgen dabei mit Blick auf die Zielstellungen der Ausrichtung am Endkundennutzen, der Realisierung von Zeitvorteilen, der Verbesserung der Qualität sowie der Minimierung des bewerteten Leistungsverzehrs. Insbesondere das zuletzt genannte Ziel ist als Anknüpfungspunkt für

Mit dem Begriff des Supply Chain Costing setzen sich u.a. auseinander: Goldbach (2002); Seuring (2001); LaLonde/Pohlen (1996). 
eine Adaption rechnungswesenrelevanter Aspekte zu interpretieren. Auch die Entwicklung des Kostenmanagement aus der traditionellen Kostenrechnung verdeutlicht die neuen Anforderungen, die an Instrumente zur Kostenidentifikation und -beeinflussung gestellt werden. Durch die Anwendung der Kostenrechnung im Supply Chain Management ergeben sich zusätzliche Anforderungen an ein unternehmensübergreifendes Kostenmanagement, die vom Supply Chain Costing zu berücksichtigen sind.

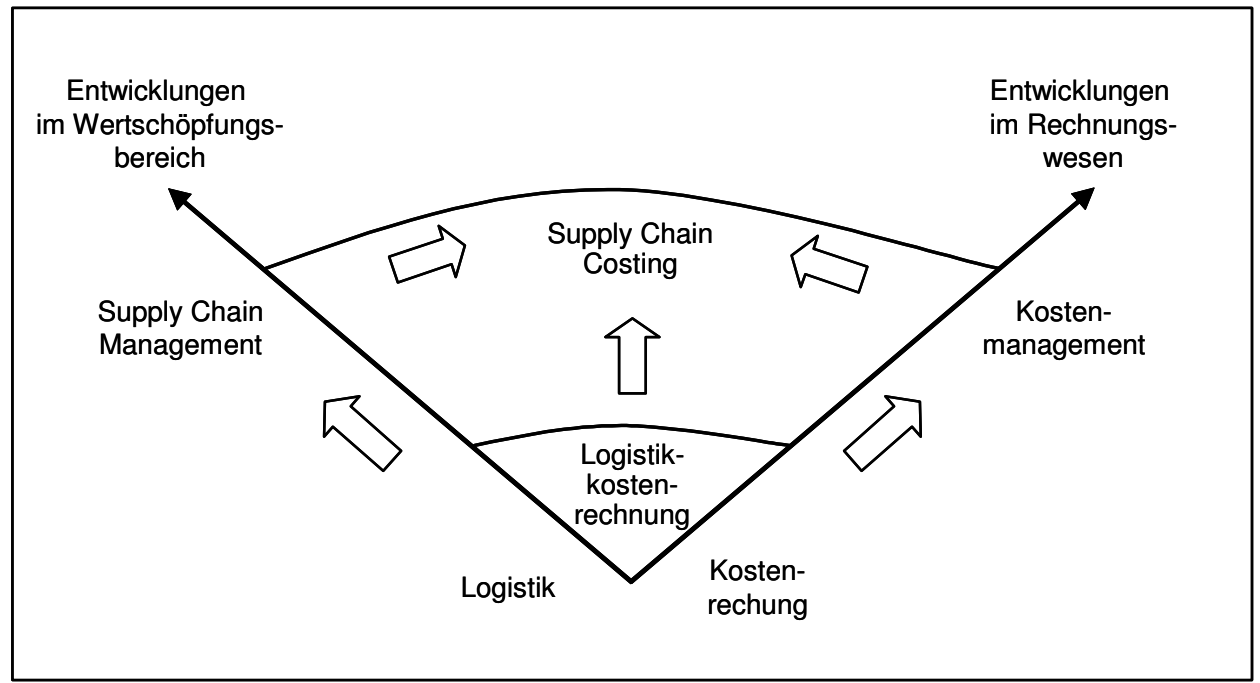

Abbildung 16: Historische Entwicklung des Supply Chain Costing

\subsection{Von der Logistik zum Supply Chain Management}

Die Logistik als unternehmerische Querschnittsfunktion ${ }^{1}$ wird in der betriebswirtschaftlichen Theorie ebenso wie in der Praxis als Referenzpunkt des Supply Chain Management gesehen. ${ }^{2}$ In ihrem Ursprung als eigenständige Teildisziplin innerhalb der Betriebswirtschaftslehre kommt der Logistik vor dem Hintergrund der stärkeren Marktorientierung und komplexer werdender Produktprogramme eine große Bedeutung in Wertschöpfungsprozessen zu. ${ }^{3}$ Dies führt zu einer zunehmenden Zusammenfassung und Spezialisierung auf material- und warenbezogene Leistungen der Transferaktivitäten. ${ }^{4}$ Auf

\footnotetext{
Vgl. Pfohl (2004a), S. 70f.

Siehe zur etymologischen Herleitung und Entwicklung des Logistikbegriffs im nichtbetriebswirtschaftlichen Gebrauch Pfohl (2004a), S. 11ff.; Bloech (1997), S. 577ff. sowie zur historischen Entwicklung der Logistik Stock/Lambert (1993), S. 19ff.

Vgl. Stock/Lambert (1993), S. 7.

4 Zu diesen Aktivitäten zählen das Transportieren, Umschlagen und Lagern (TUL-Aktivitäten). Vgl. Klaus (1999), S. 25.
} 
der Grundlage erkannter und erschlossener Rationalisierungspotenziale in den Aufgabenbereichen Transportieren, Umschlagen und Lagern (TUL) können weitere Spezialisierungsmaßnahmen bei den material- und warenflussbezogenen Dienstleistungen erreicht werden. Als unternehmensbereichs- und unternehmensübergreifende Koordinationsfunktion $^{1}$ trägt die Logistik zur Realisierung von Verbesserungspotenzialen bei, indem sie sich systematisch mit den Schnittstellen- und Integrationsproblemen auseinander setzt, die mit der Arbeitsteilung und Dislozierung einher gehen. ${ }^{2}$ Die Funktionsintegration der Logistik führt dazu, den Anspruch der ganzheitlichen und querschnittsbezogenen Gestaltung des wirtschaftlichen Handelns zu unterstützen. Insbesondere durch neue Informations- und Kommunikationstechnologien wird der Handlungsspielraum der Logistik zwischen Quelle sowie Senke erheblich erweitert. ${ }^{3}$

Die Logistik ${ }^{4}$ verfolgt im Hinblick auf die gestiegene Wettbewerbsintensität mit ihren bekannten Auswirkungen auf die Unternehmensziele sowohl Kosten- als auch Differenzierungsvorteile. ${ }^{5}$ In diesem Zusammenhang sorgt die Prozessorientierung der Logistik für eine Ausrichtung der Wertschöpfungsaktivitäten an den Bedürfnissen der Endkunden. ${ }^{6}$ Das Prozessdenken sieht die arbeitsteiligen Wertschöpfungsprozesse im Kontext interner Kunden-Lieferanten-Beziehungen, so dass dem Gedanken der Kundenorientierung sowohl intern als auch extern Rechnung getragen wird.

Die jüngste Entwicklung der betriebswirtschaftlichen Logistikkonzeption konzentriert sich auf die unternehmensübergreifende Gestaltung der Waren- und Informationsflüsse und bezieht sämtliche vor- und nachgelagerte Wertschöpfungsstufen in den Fokus der Betrachtung ein. Zunehmend gewinnen Leistungen an Bedeutung, die sich aus der gemeinsamen Betrachtung aufeinander folgender Wertschöpfungsstufen ergeben und sich am Endkunden ausrichten. Neben der unternehmensübergreifenden Koordination der Material-, Waren- und Informationsflüsse wird die kooperative Gestaltung und Integration der Geschäftsprozesse, zu denen auch Finanzflüsse sowie Eigentums- und Risikoübergänge zu zählen sind, mit allen relevanten institutionellen Wertschöpfungsakteuren als Supply Chain Management diskutiert. ${ }^{7}$

Die in der Literatur vorzufindenden Definitionsansätze des Supply Chain Management sind vielschichtig und nicht immer übereinstimmend. Als mögliche Gründe dieser Un-

Vgl. Weber (1992), S. 878ff.

Man spricht in diesem Zusammenhang von der Entstehung der Unternehmenslogistik als Gesamtsystem. Vgl. Pfohl (2004b), S. 19.

Vgl. Weber (1999), S. 8ff.; Weber (1992), S. 877..

Vgl. Weber (2002a), S. 14; Delfmann (1999), S. 46. In diesem Zusammenhang wird von Fliesssystemen gesprochen und die Logistik als "Flow Management" bezeichnet. Vgl. Klaus (1999), S. $28 \mathrm{ff}$.

Vgl. Ihde (2001), S. 21; Göpfert (1999), S. 21.

Zu diesen Managementkonzepten zählen bspw. das Kanban-, Lean Production-, Total Quality Management- und Just-in-Time-Konzept. Vgl. Aberle (2003), S. 507f.; Klaus (1999), S. 28.

7 Zum Supply Chain Management als Erweiterung der Logistikfunktion vgl. Pfohl (2004a); Krüger/Steven (2000); Bowersox/Closs (1996) sowie in einer marketingorientierten Bestandsaufnahme Min/Mentzer (1998). 
schärfe sind einerseits die Entstehung des Konzepts aus der Praxis, ${ }^{1}$ andererseits die gleichzeitige Beanspruchung einer führenden Rolle von mehreren betriebswirtschaftlichen Teildisziplinen in der Domäne des Supply Chain Management zu sehen. ${ }^{2}$ Trotz der vorherrschenden Uneinigkeit über die Definition des Supply Chain Management lässt sich ein übereinstimmendes Meinungsbild bezüglich der Zielkategorien festhalten. Für das Supply Chain Management werden auf Formalzielebene der langfristige Erhalt der Wettbewerbsfähigkeit und die Wertorientierung im Sinne einer Nutzensteigerung der Akteure der Supply Chain verfolgt. ${ }^{3}$ Auf der Ebene der Sachziele lassen sich aus der bestehenden Literatur nahezu einstimmig die Steigerung des Endkundennutzens, die Kostensenkung, die Realisierung von Zeitvorteilen sowie die Verbesserung der Qualität nennen. ${ }^{4}$ Mit der Ablösung des funktional geprägten Handelns durch unternehmensübergreifende Geschäftsprozesse verändern sich die Strukturen von Eigentumsrechten an Waren und Informationen zwischen den Unternehmen. Dies berührt ebenfalls die Sachzielebene des Supply Chain Management. Zusätzlich ergeben sich aus dem Netzwerkkontext besondere Anforderungen an Investition und Finanzierung, ${ }^{5}$ so dass der Finanzmittelfluss aktiv bei der Verwirklichung der Sachziele zu berücksichtigen ist. Dabei ist die tief greifende Bindung der Unternehmen sowie eine umfangreiche Kommunikation auf allen Ebenen der Organisationen erforderlich, um die Formalziele des Supply Chain Management zu erreichen. Realisiert werden kann dies durch die Berücksichtigung der relevanten Entscheidungstatbestände sowie der relevanten Objekte des Supply Chain Management.

Zur Erreichung dieser Ziele ist die Konfiguration des Wertschöpfungsnetzwerkes von Bedeutung. Auf Basis der logistikspezifischen Eigenschaften lassen sich unter dem Supply Chain Management-Anspruch drei im engen Zusammenhang stehende Elemente in einem konzeptionellen Bezugsrahmen identifizieren, die in Strukturen (Supply Chain Network Structure), Prozessen (Supply Chain Business Processes) und Managementkomponenten (Supply Chain Management Components) ihren Ausdruck finden und Schlüsselentscheidungen für die Akteure der Supply Chain darstellen. ${ }^{6}$ Das strukturale Element befasst sich mit der Frage, mit welchen Mitgliedern des Wertschöpfungsnetzwerkes Geschäftsprozesse zu verknüpfen sind und stellt die Grundlage des prozessualen Elements dar. ${ }^{7}$ Hier erfolgt die Identifikation der zu verknüpfenden primären Geschäftsprozesse (z.B. Beschaffung, Produktion, Distribution) sowie unterstützender Sekundärprozesse (z.B. Auftragsabwicklung, Lagerhaltung, Transport). Schliesslich befassen sich

\footnotetext{
Vgl. Göpfert (2002), S. 27; Corsten/Gössinger (2001), S. 95; Otto/Kotzab (2001), S. 159.

Der vorliegende Beitrag behandelt Supply Chain Management vor dem Hintergrund der betriebswirtschaftlichen Logistikkonzeption. Daneben behaupten auch die betriebswirtschaftlichen Disziplinen Beschaffung und Marketing das Feld für sich. Bechtel/Jayaram (1997) identifizierten fünf Denkschulen zum Supply Chain Management, die auch im deutschsprachigen Raum auf Zustimmung stoßen. Vgl. Stölzle (1999), S. 165; Kotzab (2000), S. 24ff. sowie Corsten/Gössinger (2001), S. 97.

Vgl. Christopher/Ryals (1999), S. 3.

Vgl. Mentzer u.a. (2001), S. 15; Kotzab (2000), S. 34; Stölzle (1999), S. $163 f$.

Vgl. Hofmann/Elbert (2004), S. $96 f$.

Vgl. Lambert/Cooper (2000), S. 69.

Vgl. Sydow (2002), S. 11.
} 
die Managementkomponenten mit der Festlegung des inhaltlichen Ausmaßes und der Intensität der Integration der Geschäftsprozesse (z.B. im Hinblick auf Planung, Controlling, Managementmethoden). ${ }^{1}$ Die Entscheidungstatbestände des konzeptionellen Bezugrahmens bilden auch in Bezug auf die Gesamtheit aller Steuerungsmaßnahmen zur Beeinflussung der Kosten die Substanz, auf der ein Kostenmanagement in Supply Chains aufgebaut werden kann. ${ }^{2}$

Bei den Objekten in Supply Chains handelt es sich um die aus der Logistikkonzeption stammenden materiellen Flüsse von Waren (z.B. Rohstoffe, Vorprodukte, Module, Fertigteile und Endprodukte) sowie um die vorauseilenden, synchron gekoppelten oder nachlaufenden Informationsflüsse, ergänzt um den unternehmensübergreifenden Übergang der Finanzmittel und "Eigentumsrechte". ${ }^{3}$ Dies unterstreicht den Integrations- und Koordinationszusammenhang, dem die Managementforschung eine zunehmende Aufmerksamkeit schenkt. Management versteht sich als reflexive Gestaltung und Steuerung sozialer Systeme. $\mathrm{Zu}$ der operativen Durchführung der erweiterten Logistikleistungen treten somit auch strategische Aspekte hinzu. Es geht nicht nur um die Steuerung der relevanten Objektflüsse, sondern auch um die Entwicklung von Funktional-, Geschäftsfeld- und Unternehmensstrategien. Dem Supply Chain Management kommt dabei die Aufgabe zu, die Gestaltung und Handhabung zwischenbetrieblicher bzw. leistungsübergreifender Zusammenhänge nicht an den Unternehmensgrenzen enden zu lassen, ${ }^{4}$ wobei der Ermittlung und Verrechnung von Kosten im Netzwerkkontext eine besondere Rolle zukommt. $^{5}$

Als Zwischenfazit kann konstatiert werden: Das Oberziel des Supply Chain Management besteht in der Sicherung der Wettbewerbsfähigkeit bei Minimierung des Resourceneinsatzes. Ferner handelt es sich um einen strategischen, kooperationsorientierten und unternehmensübergreifenden Managementansatz, der über eine Prozessintegration zu einer Verbesserung der logistischen Aufgabenwahrnehmung führt und dabei alle Waren-, Güter-, Informations- und Finanzflüsse auf den fokussierten Stufen der Supply Chain berücksichtigt. ${ }^{6}$ Die Ausrichtung der relevanten Aktivitäten erfolgt beim Supply Chain Management konsequent an den Bedürfnissen der Endverbraucher. ${ }^{7}$ Aus der Verbesserung der Wettbewerbsfähigkeit und der Profitabilität sowie aufgrund der zunehmenden Strategieorientierung erwächst für das Supply Chain Management der Bedarf nach Ansätzen zur langfristigen unternehmensübergreifenden Kostenbeeinflussung.

Vgl. Lambert/Cooper (2000), S. 69ff.; Stölzle (2002b), S. 285f.; Stölzle (1999), S. 11.

Diese Herleitung erfolgt in Analogie zur Eigenständigkeit des Supply Chain Controlling. Vgl. Stölzle (2002b), S. 286

Vgl. Pfohl/Hofmann/Elbert (2003), S. 15ff

Vgl. Sydow (2002), S. 10.

Vgl. Sydow (2002), S. 11.

Vgl. Kotzab (2000), S. 27

Vgl. Stölzle (2002a), S. 10; Stölzle (1999), S. 164ff. 


\subsection{Von der Kostenrechnung zum Kostenmanagement}

Als Instrument des internen Rechnungswesens trägt die Kostenrechnung zentral zum Verständnis der Funktionsweisen und zur Durchführung der wirtschaftlichen Leistungserstellung in Unternehmen bei. Dabei ist es nicht verwunderlich, dass ein einheitlicher Entwicklungsstand der Konzepte, Instrumente, Systeme und Anwendungen in Theorie und Praxis nicht zu identifizieren ist. Generell umfassen die verschiedenen Kostenrechnungssysteme die Teilbereiche Kostenarten-, Kostenstellen- und Kostenträgerrechnung. ${ }^{1}$ $\mathrm{Zu}$ den grundsätzlichen Aufgaben der Kostenrechnung gehören die Erfassung und Zurechnung der Kosten im Sinne des bewerteten sachzielbezogenen Güterverbrauchs einer Periode, der bei der betrieblichen Leistungserstellung und -verwertung entsteht. ${ }^{2}$

In einer häufig anzutreffenden, vergleichsweise einfachen Strukturierung lassen sich drei Hauptfunktionen der Kostenrechnung unterscheiden: Dokumentations-, Planungs- und Kontrollfunktionen. ${ }^{3}$ Mit der regelmässigen Erfassung der Kosten, die im Leistungserstellungsprozess entstehen, sowie der Zuteilung dieser Kosten auf Kostenstellen und Kostenträger stellt die Kostenrechnung Informationen über die Kostensituation im Speziellen sowie den Erfolg eines Unternehmens im Allgemeinen bereit (Dokumentationsfunktion), dient der Kontrolle der Wirtschaftlichkeit von Leistungserstellungsprozessen (Kontrollfunktion) und gibt Anhaltspunkte für den künftigen Ressourceneinsatz (Planungsfunktion). ${ }^{4}$ Da sich damit zentrale Entscheidungen auf Funktions-, Bereichs- oder Unternehmensebene vollziehen lassen (z.B. über den Fremdbezug oder die Eigenerstellung von Leistungen), handelt es sich bei der Kostenrechnung primär um ein internes Führungsunterstützungs- und Informationssystem. ${ }^{5}$

Traditionelle Ansätze der Kostenrechnung verlieren jedoch in der jüngeren Vergangenheit aufgrund unterschiedlicher Entwicklungstendenzen zunehmend an Bedeutung. Einerseits treten mit der zunehmenden Strategieorientierung, die zu einem wesentlich längeren Planungshorizont führt, Forderungen nach einer stärkeren Zukunftsorientierung und einer höheren Kostentransparenz auf. ${ }^{6}$ Zwar können mit Hilfe der Plankostenrechnung Kosten und Erlöse für eine bestimmte Planperiode im voraus berechnet werden, ${ }^{7}$ die für die Strategiewahl schwer quantifizierbaren Größen wie Kundenzufriedenheit, Qualität, Flexibilität und Zeitvorteile finden jedoch durch die klassischen Kostenrech-

Vgl. Coenenberg (2003), S. 12.

Vgl. Hummel/Männel (1986), S. 11.

Vgl. Fischer (2002), S. 49; Freidank (1997), S. 93

Neben diesen Hauptfunktionen lassen sich auch die Initiativfunktion zur Identifikation von Kostentreibern, die Bewertungsfunktion zur Beurteilung von Handlungsalternativen und die Beobachtungsfunktion zur Rückmeldung von Kostensenkungen als weitere Funktionen der Kostenrechnung für das Kostenmanagement nennen. Vgl. Fischer (2002), S. 51

Vgl. Schöttler/Spulak (1989), S. 45.

Vgl. Kajüter (2005), S. 85.

Plankostenrechnungssysteme lassen sich wie die übrigen Kostenrechnungssysteme in eine Kostenarten-, eine Kostenstellen- und eine Kostenträgerrechnung untergliedern. Vgl. Joose-Sachse (2000), S. 171. 
nungssysteme keine ausreichende Berücksichtigung. ${ }^{1}$ Der Einsatz kapitalintensiver Technologien wirkt sich zudem mit einer Verschiebung von Einzelkosten hin zu Gemeinkosten auf die Kostenstruktur aus. ${ }^{2}$ Daraus resultieren Veränderungen in der Kostenverrechnung. Denn die anteilig höheren Gemeinkosten führen zu steigenden $\mathrm{Zu}$ schlagssätzen in der Kostenstellenrechnung. Damit geht eine nicht adäquate Abbildung der indirekten Leistungsbereiche in der Kalkulation einher.

Als Konsequenz der beschriebenen Entwicklungen und unter Berücksichtigung der Marktausrichtung von Wertschöpfungsnetzwerken zeigt sich, dass die traditionelle Kostenrechnung ohne Anpassungen die relevanten Funktionen nur unzureichend erfüllt. ${ }^{3} \mathrm{Um}$ Rationalisierungspotenziale nutzen zu können, hat sich die Kostenrechnung den veränderten Einflüssen anzupassen. Die Entwicklung der Kostenrechnung als Anbieter primär vergangenheitsorientierter Informationen zum Kostenmanagement dient der aktiven und zukunftsgerichteten Kostenbeeinflussung. Denn die Steuerung und Beeinflussung der Kosten sowie die Verbesserung der relativen Kostenposition zur anhaltenden Steigerung der Wettbewerbsfähigkeit nehmen eine Schlüsselrolle im Management ein.

Unter diesen Gesichtspunkten umfasst das Kostenmanagement die Gesamtheit aller Steuerungsmaßnahmen, die der frühzeitigen und antizipativen Beeinflussung von Kostenstrukturen und Kostenverhalten sowie der Senkung des Kostenniveaus dienen. ${ }^{4} \mathrm{Zu}$ den Gestaltungsobjekten des Kostenmanagements lassen sich die Senkung des Kostenniveaus, sowie die Optimierung der Kostenstruktur und des Kostenverlaufs nennen. Die daraus abgeleiteten Ziele sind interdependent, da die Kostenstruktur Einfluss auf den Kostenverlauf hat und sich beide auf das Kostenniveau auswirken. Ferner soll die Betonung des Kostenbewusstseins bei den Mitarbeitern sowie die Erhöhung der Kostentransparenz zur Verbesserung der Kostensituation in Unternehmen beitragen. ${ }^{5}$

Das Management der Kostenstruktur setzt an dem Missverhältnis bzw. der Unausgewogenheit einzelner Kostenkategorien an. Der Fokus liegt auf der Zusammensetzung von fixen und variablen Kosten bzw. Gemein- und Einzelkosten. Kostenstrukturen mit einem hohen Anteil an Fixkosten und einem geringen variablen Anteil werden als unvorteilhaft angesehen, weil sie aufgrund ihrer Kostenhöhe bei gegebener Kapazität unabhängig von der Ausbringungsmenge konstant bleiben. Beim Aufbau von Fixkosten ist darauf zu achten, dass durch steigende Mengen eine Fixkostendegression genutzt werden kann. ${ }^{6}$ Ebenso wirken sich große Gemeinkostenblöcke nachteilig auf eine verursachungsgerechte

Vgl. Brede (1993), S. 338. Diese Entwicklung spiegelt sich auch in der wissenschaftlichen Diskussion zur Balanced Scorcard wieder. Vgl. zur Balanced Scorecard im Allgemeinen Kaplan/Norton (1997) sowie im Supply Chain-Kontext Stölzle/Heusler/Karrer (2001).

Vgl. Coenenberg (2003), S. 205ff.

Vgl. Corsten (2001), S. 2.

Vgl. Kajüter (2000), S. 115ff.; Dellmann/Franz (1994), S. 17; Brede (1993), S. 2.

Vgl. Kajüter (2005), S. 85. Bereits früh wurden diese Gestaltungsobjekte des Kostenmanagements im deutschsprachigen Raum von Reiß/Corsten (1990) vorgestellt.

6 Vgl. Corsten (2001), S. 14 
Zurechnung von Kosten auf Produkte sowie Prozesse aus und erschweren die Steuerung der Unternehmensaktivitäten. ${ }^{1}$

Beim Kostenverlaufsmanagement steht die Beeinflussung des Kostenverhaltens in Abhängigkeit der Beschäftigung pro erstellter Leistungseinheit im Vordergrund. Die Grundformen der Kostenverläufe sind proportional, progressiv oder degressiv. Der proportionale Kostenverlauf ermöglicht ein stetiges und gleichmäßiges Kostenverhalten. Bei einem progressiven Kostenverlauf nehmen die Gesamtkosten mit steigender Menge je Stück zu. Gründe hierfür können bspw. steigende Koordinationskosten sein. Degressive Kostenverläufe hingegen sind durch abnehmende Grenzkosten bei steigender Ausbringungsmenge gekennzeichnet. Ermöglicht werden Kostendegressionen durch die Realisierung von Skaleneffekten oder durch Spezialisierung (z.B. sinkende Materialbeschaffungskosten beim Vorliegen von Mengenrabatten). ${ }^{2}$ Auch Lernprozesse in Funktionsbereichen wie Beschaffung, Produktion und Distribution tragen hierzu bei. In Summe steht die Förderung degressiver bzw. die Vermeidung progressiver Kostenverläufe im Vordergrund, um das Kostenverhalten im Sinne des Kostenmanagement positiv zu beeinflussen.

Mit dem Kostenniveaumanagement soll die allgemeine Kostenhöhe gesenkt werden. Dies kann durch Rationalisierungsmaßnahmen bspw. in Form eines Gemeinkostenmanagement erfolgen. Dabei ergeben sich Ansatzpunkte sowohl bei der Mengen- als auch bei der Wertkomponente. Die Mengenkomponente setzt unmittelbar an den Verbrauchsmengen der eingesetzten Produktionsfaktoren an. Mit Hilfe von Wertanalysen, mit der die existierende Produkte und Verfahren verbessert werden sollen, kann z.B. die Senkung der Funktions-, Produktions- und Materialkosten erzielt werden. Eine Ablaufanalyse dient als weitere Maßnahmen zur Senkung des Kostenniveaus. Hierzu zählen die Vermeidung von Doppelarbeit und Medienbrüchen sowie die Reduzierung der Durchlaufzeiten. Die Wertkomponente setzt am Wertgerüst der Kosten an und unterstützt die kostenorientierte Standortwahl, erleichtert Entscheidungen über Fremdbezug oder Eigenerstellung und ermöglicht eine kostenminimale Lieferantenauswahl. ${ }^{3}$

Neben den Gestaltungsobjekten der Kostenstruktur, des Kostenverlaufs und des Kostenniveaus kann in einer alternativen Betrachtungsweise das Kostenmanagement an der langfristigen Verbesserung der Kostenposition zur dauerhaften Sicherung der Wettbewerbsfähigkeit ansetzen. ${ }^{4}$ Kostenmanagement greift hierbei an den Beziehungszusammenhängen der Leistungserstellung an. ${ }^{5} \mathrm{Zu}$ den Gestaltungsobjekten gehören hierbei die an Kundenwünschen ausgerichteten Produkte, die zur Erstellung der Produkte notwendigen Prozesse und die für die Prozesse benötigten Produktionsfaktoren (Arbeitskraft, Ma-

Vgl. Coenenberg (2003), S. 54f.

Weitere Einflussfaktoren auf den degressiven Kostenverlauf stellen die Verringerung der Produktionskoeffizienten dar. Vgl. Reiß/Corsten (1990), S. 391.

Vgl. Reiß/Corsten (1990), S. 390f.

Vgl. Männel (1993), S. 210ff.

Vgl. Franz/Kajüter (1997), S. 12. 
schinen, Betriebsstoffe). ${ }^{1}$ In Abhängigkeit der Eigentumsverhältnisse an den Produktionsfaktoren lässt sich eine interne und externe Einteilung aus individueller Unternehmenssicht vornehmen. Diese Sichtweise wird im Zuge der Vernetzung von Unternehmen zunehmend aufgelöst und durch eine unternehmensübergreifende Perspektive ersetzt.

Der strategische Anspruch des Kostenmanagement manifestiert sich in der Erzielung nachhaltiger Kostensenkungspotenziale im dynamischen Wettbewerb. Für Verbesserungen der Produkt- und Prozessstrukturen reichen die Informationen der Kostenrechnung allein nicht aus. $\mathrm{Zu}$ den strategischen Aufgaben des Kostenmanagement gehören die Abschätzung und Bewertung von Kostenimplikationen von Strategieentscheidungen sowie die Identifikation von Einflussgrössen auf die strategische Kostenposition. Weiterhin umfasst es die handlungsorientierte Ermittlung und Darstellung von Kosten sowie die konkurrenzbezogene Analyse und Vorgabe von Kosten in Wertschöpfungsnetzwerken. ${ }^{2}$ Neben der Verfolgung einer dauerhaften Wettbewerbsfähigkeit wirken auch gilt es, operative Verbesserungen des gegenwärtigen bewerteten Ressourcenverzehrs zu beleuchten. Für diese operative Dimension des Kostenmanagement stellen die Funktionen der Kostenrechnung eine Voraussetzung dar, um innerhalb der gegebenen Produkt- und Prozessgestaltung die Effizienz zu steigern. ${ }^{3} \mathrm{Zu}$ den Aufgaben des operativen Kostenmanagement zählen die Auswertung, Planung und Vorgabe der Kostenimplikationen operativer Entscheidungen.

Anhand dieses Abschnittes wurde deutlich, dass dem Kostenmanagement vielfältige Aufgaben zukommen, um den Herausforderungen in der unternehmerischen Praxis zu begegnen. Neben der Kostenstruktur, dem Kostenverlauf und dem Kostenniveau stellen Produkte, Prozesse sowie interne und externe Produktionsfaktoren die Gestaltungsobjekte des Kostenmanagement dar.

\subsection{Logistikkostenrechnung als Referenzpunkt des Kostenma- nagement in Supply Chains}

Unter Logistikkostenrechnung ist die Wahrnehmung von Kostenrechnungsaufgaben im Logistikbereich des Unternehmens zu verstehen. Sie ist als Subsystem des gesamten Rechnungswesens aufzufassen. Eine besondere Rolle spielt dabei der Koordinationsanspruch, der für die Logistik im Leistungsbereich und für das Kostenmanagement im Führungsbereich als prägend gilt. ${ }^{4}$ Logistikkosten entstehen in ihrer absoluten Höhe und in ihrer Aufteilung in den verrichtungsspezifischen Logistiksubsystemen Auftragsabwick-

Franz/Kajüter (2002) sprechen in diesem Zusammenhang nicht von Produktionsfaktoren, sondern von Ressourcen. Vgl. Franz/Kajüter (2002), S. 24-27.

Vgl. Dellmann/Franz (1994), S. 18.

Vgl. Franz/Kajüter (2000), S. 117.

Vgl. Abschnitt 2.1. 
lung, Lagerhaltung, Lagerhaus, Verpackung und Transport ${ }^{1}$ sowie in den phasenspezifischen Subsystemen Beschaffung, Produktion, Distribution und Entsorgung. ${ }^{2}$ Die "klassische" Auslegung von Kostenrechnungssystemen mit ihren Teilbereichen Kostenarten-, Kostenstellen- und Kostenträgerrechnung ist aufgrund der Fertigungsorientierung auf den speziellen Informationsbedarf der Logistik nur unzureichend ausgerichtet. ${ }^{3}$ Im Folgenden wird dargestellt, welche Herausforderungen bei einer logistikorientierten Kostenarten-, Kostenstellen- und Kostenträgerrechnung zu bewältigen sind.

Die Gliederung, Erfassung und Zurechnung der für die betrieblichen Leistungen angefallenen Kosten erfolgt im Rahmen der Kostenartenrechnung in Einzel- und Gemeinkosten für die Kostenstellen- und die Kostenträgerrechung. ${ }^{4}$ Im Logistikkontext sind Einzelkosten häufig Fremdleistungskosten, die Produkten oder deren Vorstufen zuzurechnen sind. ${ }^{5}$ Logistik-Gemeinkosten dagegen fallen auf Kostenstellen an, deren Leistungen wiederum an andere Kostenstellen verrechnet werden. Sie können sowohl Fremdleistungskosten (z.B. Fremdvergabe des innerbetrieblichen Transports) als auch Eigenleistungskosten sein (z.B. selbst erbrachter innerbetrieblicher Transport). Die Verrechnung von Logistikkosten wird zunächst dadurch erschwert, dass viele Logistikaktivitäten ohne korrespondierende Zahlungen ablaufen (z.B. Lagervorgänge) und deshalb keinen Bezug zum Marktwert der erbrachten Leistungen aufweisen. Zudem weisen Logistikaktivitäten häufig Merkmale der Kuppelproduktion auf bzw. besitzen einen Verbundcharakter. Damit ist die Erfassung der Logistikkosten in der Kostenartenrechnung durch vielfältige, im Einzelfall festzulegende Gestaltungsoptionen gekennzeichnet. So werden Personal, Anlagen und Energie über die gesamte Wertschöpfungskette eingesetzt und lassen sich den Kostenstellen nur schwer überschneidungsfrei zuordnen. ${ }^{6} \mathrm{Zu}$ diesen Abbildungsproblemen trägt auch dazu bei, dass Logistikleistungen aufgrund ihres immateriellen Charakters schwer erfassbar sind. ${ }^{7}$

Die verursachungsgerechte Verrechnung von Logistikkosten auf Kostenstellen stellt aufgrund der unterschiedlichen Möglichkeiten der Festlegung von Verrechnungspreisen ein zentrales Problem dar. Kostenstellen für logistische Leistungsbereiche finden sich zumeist in kunden- und lieferantennahen Distributions- bzw. Beschaffungsbereichen. Beispiele sind etwa der eigene Fuhrpark für Warenauslieferungen. Dagegen finden sich innerhalb der Unternehmen und besonders in der Produktion nur wenige Logistikkostenstellen wie etwa "interner Transport". ${ }^{8}$ Gelingt es, die Logistik in der Kostenstellenrechnung zu berücksichtigen, werden eine größere Transparenz über material- und warenflussbezogene Dienstleistungen erzielt sowie die Identifikation und Zuordnung von Lo-

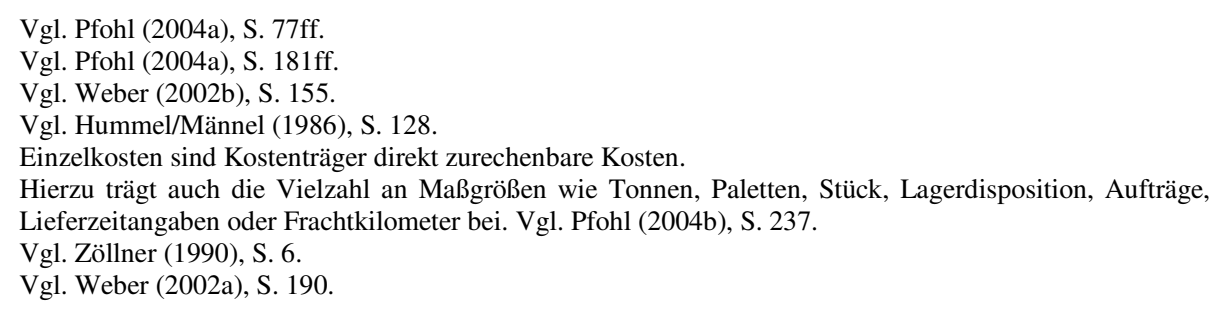


gistikkosten erleichtert. Dabei führt auf der Ebene der Logistikkostenstellen die Unterscheidung von Bereitschafts- und Leistungskosten zu Transparenz über das Ausmass einer leistungsabhängigen Anpassung von Logistikkosten. Zudem ermöglichen Logistikkostenstellen eine Kostenkontrolle der logistischen Aufgabenwahrnehmung. ${ }^{1}$

Die Aufgabe der Kostenträgerrechnung liegt in der Ermittlung der Selbstkosten der angebotenen Produkte bzw. Dienstleistungen. ${ }^{2}$ Mit Hilfe von Kalkulationssätzen werden die auf den Endkostenstellen gesammelten Gemeinkosten gemäß anteiliger Inanspruchnahme der jeweiligen Kapazität auf die Kostenträger verrechnet verteilt und mit den Einzelkosten zusammengeführt. ${ }^{3}$ Auch die im Leistungserstellungsprozess anfallenden Logistikkosten müssen Kostenträgern zugerechnet werden. Zunächst gilt es, die Kostenwirkung der Logistik auf einzelne Wertschöpfungsaktivitäten sichtbar zu machen, damit logistikspezifische Besonderheiten bestimmt werden können (z.B Mindesttransportlosgrößen, Kostenzuschläge in der Transportplanung). Weiterhin bildet die Zurechenbarkeit der anfallenden Kosten auf einzelne Kunden bzw. Unternehmensbereiche eine Voraussetzung für die Formulierung differenzierter Logistikstrategien. Schließlich unterstützt eine Logistikkostenträgerrechnung eine differenzierte Preiskalkulation für die abzusetzenden Leistungen nach Massgabe der Inanspruchnahme logistischer Kapazitäten.

Der Beitrag der Logistikkostenrechnung ist von den Zwecken, die man mit ihr verfolgt, sowie von der Unternehmenssituation, in die ein solches Rechnungssystem eingebettet werden muss, abhängig. Der differenzierte Ausweis von Logistikkosten zur Steigerung der Transparenz des Logistikbereichs ist dabei sicherlich als ein Ziel zu bezeichnen. Eine Entscheidung über den Differenzierungsgrad befasst sich zum einen mit der Frage, wie detailliert die unternehmensindividuelle Logistikkostenrechnung ausgestaltet sein soll ("Differenzierung in die Tiefe"). Zum anderen ist zu beantworten, ob die Kostenrechnung auf vor- und/oder nachgelagerte Wertschöpfungsstufen ausgedehnt werden soll ("Differenzierung in der Breite"). Bezüglich des ersten Punkts ist zu beobachten, dass der Trend hin zu einer "schlanken" Logistikkostenrechnung geht. ${ }^{4}$ Damit ist ein auf die spezifischen Zwecke der Logistikkosten ausgerichtetes Kostenrechnungssystem gemeint. $\mathrm{Zu}$ begründen ist dies damit, dass eine hoch differenzierte Kostenrechnung weder den Anforderungen vorausschauender Investitionsrechnungen genügt, noch dem Informationsbedarf der Strategieplanung entspricht. Weiterhin scheint das Ziel einer hohen Transparenz bzw. Nachvollziehbarkeit der Kostenerfassung gegenüber dem einer differenzierten Kostenaufschlüsselung aus Sicht der Entscheidungsträger prioritär. Damit steht eher das Management der Logistikkosten als die Logistikkostenrechnung im Zentrum des Interesses. Das Management der Logistikkosten scheint insbesondere im Supply Chain-

Vgl. Weber (2002a), S. 190.

Vgl. Joos-Sachse (2002), S. 98

Vgl. Schweitzer/Küpper (2003), S. 155ff. Zu den verbreiteten Verfahren der Umlegung von fixen Gemeinkosten zählen die Lohnzuschlagskalkulation und die Verrechnungssatzkalkulation. Vgl. Weber (1997), S. 80ff.

4 Vgl. Pfohl (2004b), S. 239f. 
Umfeld von Bedeutung. Bezogen auf den zweiten Punkt herrschen in der Praxis derzeit meist so genannte unternehmensindividuelle "Kostenrechnungsinseln" vor. Vor- und nachgelagerte Wertschöpfungssysteme sind aus Sicht des jeweiligen Rechnungssystems nicht miteinander verknüpft. Logistikkosten, die vor allem zwischen den einzelnen Wertschöpfungsstufen anfallen, lassen sich nicht eindeutig dem einen oder anderen Supply Chain-Akteur zuordnen. Der Informationsbedarf aus einer unternehmensübergreifenden Perspektive wird nur unzureichend erfüllt.

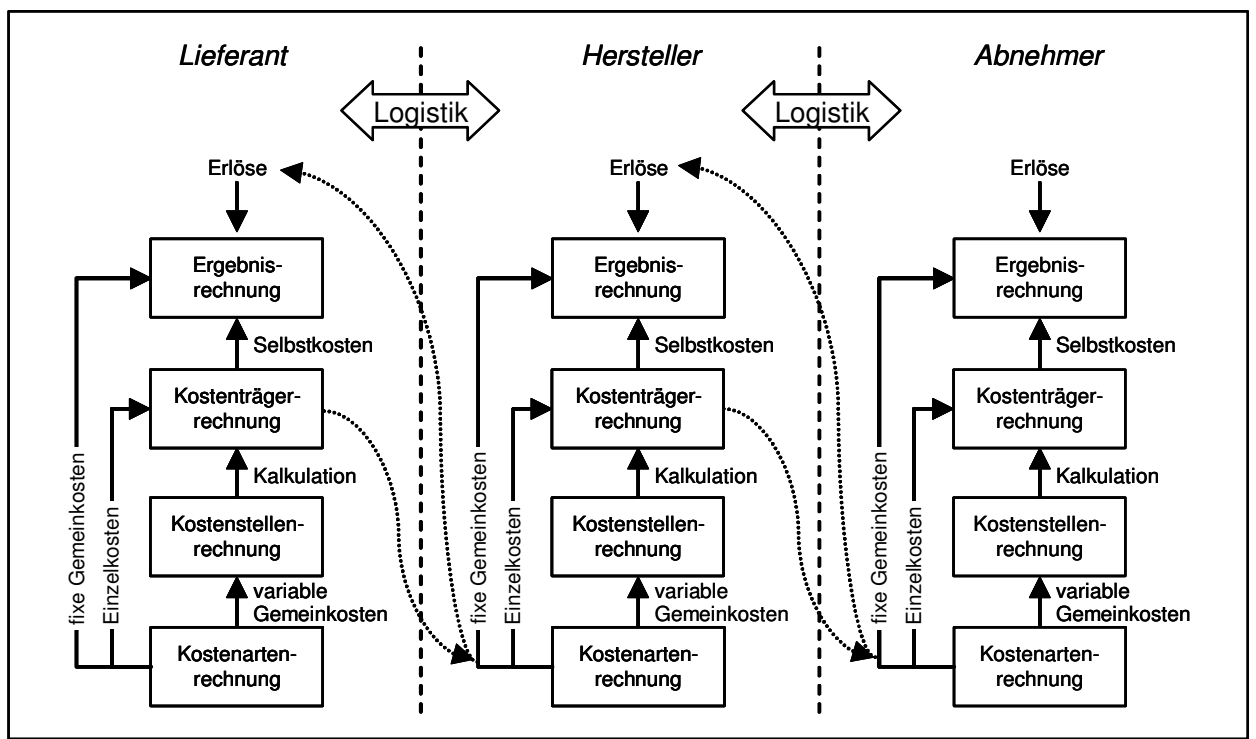

Abbildung 17: Kostenrechnungsinseln in Supply Chains

Ein "schlankes" Logistikkostenmanagement, das auf die vor- und nachgelagerten Wertschöpfungsstufen ausgedehnt wird und über eine hohe Akzeptanz verfügt, kann als Referenzpunkt für das Kostenmanagement in Supply Chains betrachtet werden. Ein unternehmensübergreifendes Kostenmanagement kommt dem Querschnittscharakter der Logistik und dem übergreifenden Charakter des Supply Chain Management am besten entgegen, stellt aber hohe Anforderungen an die Informationsverarbeitung sowie an die Dokumentations-, Planungs- und Kontroll-Kapazitäten der historisch gewachsenen Kostenrechnungsstrukturen der beteiligten Unternehmen. Es setzt den Aufbau eines standardisierten Kostenrechnungssystems aus einer netzwerkweiten Grundrechnung und verschiedene zweckgerichtete Auswertungsrechnungen (z.B. im Hinblick auf Endprodukte, den gesamten Ressourcenverbrauch oder die Prozesseffizienz) voraus. Realisieren lassen sich solche übergreifenden Systeme nur dann, wenn es zu einer Harmonisierung der unternehmensbezogenen Rechnungssysteme kommt. Die Gestaltungsalternativen der Rechnungssysteme in einer Supply Chain können dabei sehr unterschiedlich ausfallen. Bei einer Harmonisierung über alle Supply Chain-Akteure hinweg soll von einem voll integrierten Kostenrechnungssystem gesprochen werden. Aufgrund der Beteiligung an 
verschiedenen Wertschöpfungsnetzwerken ist ohne einen branchenübergreifenden Standard ein voll integrierendes Rechnungssystem allerdings als Vision zu werten. Vielmehr erfolgt im Rahmen eines partiell integrierten Rechnungssystems die Harmonisierung des Kostenmanagement zwischen einzelnen Akteuren der Supply Chain, bspw. zwischen Lieferanten und Hersteller. Einzelne sensible Rechnungswesenbereiche wie die Ergebnisrechnung können dabei ausgenommen werden. Eine lediglich koordinierende Funktion mit den Rechnungssystemen von Supply Chain Akteuren zu, wenn eine Abstimmung ausbleibt und nicht-standardisierte Kosteninformationen fallweise über Kenngrößen ausgetauscht werden.

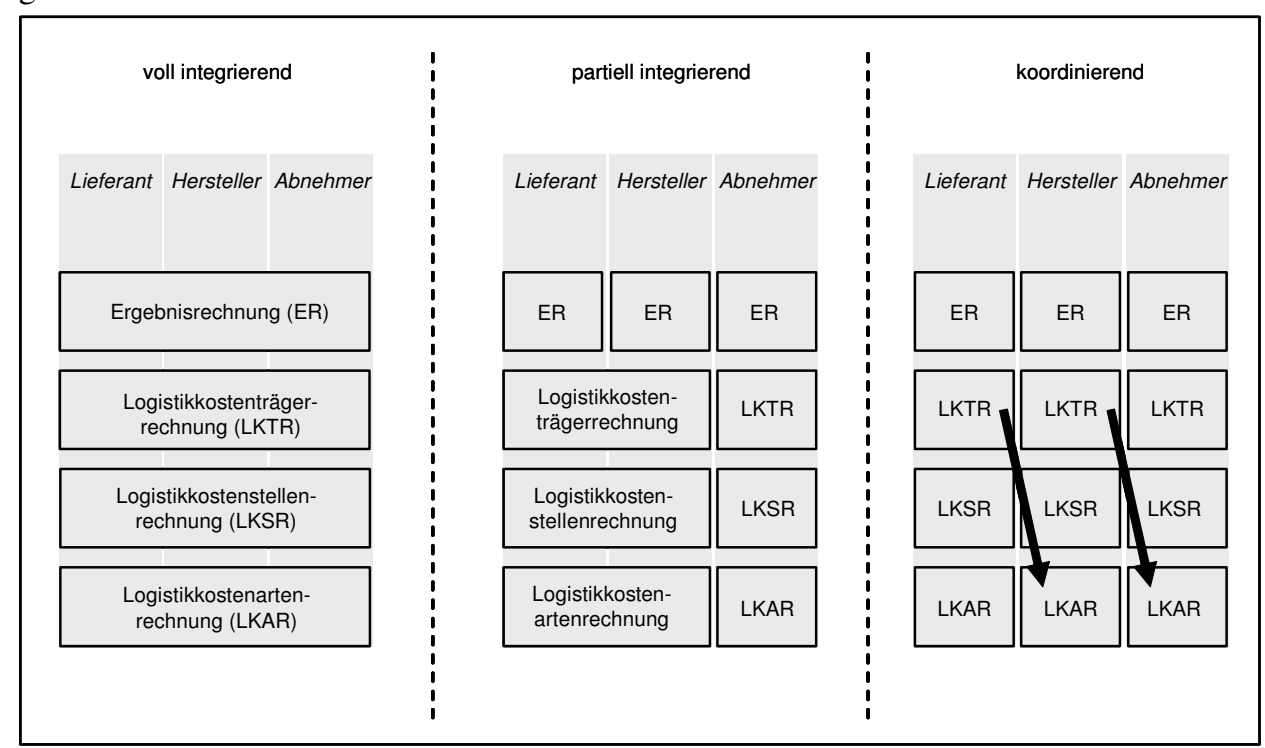

Abbildung 18: Generische Gestaltungsalternativen für unternehmensübergreifende Logistikkostenrechnungssysteme

\subsection{Anforderungen an das Kostenmanagement in Supply Chains}

Unternehmen erreichen mit Hilfe der Kostenrechnung eine erhöhte interne Kostentransparenz bei den eigenverantwortlich erstellten Leistungen. Neue Herausforderungen ergeben sich jedoch im Hinblick auf die Kostentransparenz im Rahmen der Zusammenarbeit mit Supply Chain-Akteuren. Denn erstens wird in jedem Unternehmen die interne Kostenerfassung und -verrechnung einem spezifisch ausgelegten Rechnungssystem realisiert. Beispielsweise sind bekannte Gemeinkostenzuschlagssätze ebenso wie die Wahl 
von Bezugsgrößen das Ergebnis einer rein unternehmensbezogen ausgerichteten Kostenrechnung. ${ }^{1}$ Zweitens bedarf es für eine (nur annähernd) verursachungsgerechte Kostenzuordnung über mehrere Unternehmen Abstimmungsmechanismen über die Modalitäten der unternehmensübergreifenden Kostenverrechnung. Dabei zeigt sich in Anlehnung an den Verlauf der steigenden Auftragsschwankungen flussaufwärts in einer Supply Chain die Verstärkung von Kostenschwankungen, die zu zunehmenden Verzerrungen bei einer unternehmensübergreifend angelegten Ergebnisrechnung ${ }^{2}$ und Kalkulation ${ }^{3}$ führen. Diese Schwankungen wirken sich negativ auf die Informationsqualität für unternehmensbezogene Entscheidungen aus. ${ }^{4}$ Umgekehrt führt dies zu einem entgegen gesetzten Aufschaukeln von Kalkulationsungenauigkeiten flussabwärts. Preise bzw. Verrechnungssätze werden beispielsweise zur Kompensation von Unsicherheiten überhöht festgelegt. Mit der Übertragung von nicht-standardisierten Kosteninformationen ist demnach eine unzureichende Güte von Kostenaussagen verbunden. Dies beeinträchtigt die Entscheidungsqualität ebenso wie die strategische Aufgabe des Kostenmanagement, nachhaltige Kostensenkungspotenziale zu realisieren (vgl. Abbildung 19). Zudem kann keine durchgängige Kontrolle der Prozesseffizienz über die verschiedenen Wertschöpfungsstufen erfolgen.

Im Rahmen des Supply Chain Management besteht die Herausforderung für das Kostenmanagement darin, die enge Zusammenarbeit von rechtlich und wirtschaftlich selbständigen Unternehmen zu unterstützen und den gemeinsamen erfolgreichen Auftritt am Markt zu sichern. ${ }^{5}$ Auf Basis der unternehmensbezogenen Ausrichtung sind damit eine gemeinsame Supply Chain-Strategiewahl und eine gemeinsame Einigung auf daraus abzuleitende Ziele verbunden.

Durch eine Prozessintegration konkurrieren nicht mehr einzelne Unternehmen, sondern vielmehr verschiedene Supply Chains miteinander. ${ }^{6}$ Dabei wird neben der Strategie der Leistungsführerschaft insbesondere die Kostenführerschaft zur Erzielung von Wettbewerbsvorteilen angestrebt. ${ }^{7}$ Mit einer Ergebnisverbesserung aufgrund einer Kostensenkung oder einer Erlössteigerung verfolgen die Akteure sowohl die Wertsteigerung des eigenen Unternehmens als auch der gesamten Supply Chain. ${ }^{8}$ Die daraus abgeleiteten

\footnotetext{
Vgl. Cooper (1988), S. 46

Im Rahmen der Kostenträgerzeitrechnung bzw. Ergebnisrechnung werden die erstellten Leistungen einer Periode den entstandenen Kosten gegenüber gestellt. Vgl. Jórasz (1996), S. 167.

3 Mit Hilfe der Kostenträgerergebnisrechnung bzw. Kalkulation wird der Erfolg einzelner Produkte und Produktgruppen ermittelt, in dem die diesen Produkten zuordenbaren Kosten und Leistungen einer Periode bestimmt werden. Vgl. Hummel/Männel (1986), S. 167.

4 Der Bullwhip-Effekt wird durch das Phänomen beschrieben, dass bei jeweils lokal eingeschränkten Informationen kleinere Schwankungen der Kundenbedarfe auf jeder weiteren Stufe der Supply Chain zu einer immer weiter ansteigenden Streuung der Bedarfsmengen führen. Vgl. Forrester (1958), S. $37 \mathrm{ff}$.

Vgl. Weber (2002b), S. 185f.

Vgl. Lambert/Cooper (2000), S. 65.

Vgl. Porter (1999), S. 70ff.

Zur Ausrichtung der Unternehmens- und Supply Chain-Aktivitäten im Rahmen der wertorientierten Steuerung vgl. Stölzle/Karrer (2004).
} 
Verstärkung von Kostenschwankungen (flussaufwärts)

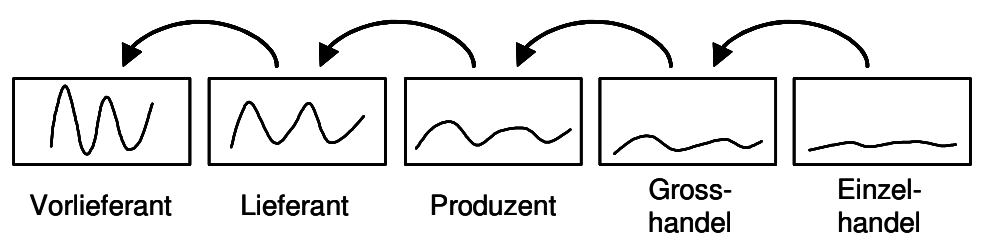

Aufschaukeln von Kalkulationsungenauigkeiten (flussabwärts)

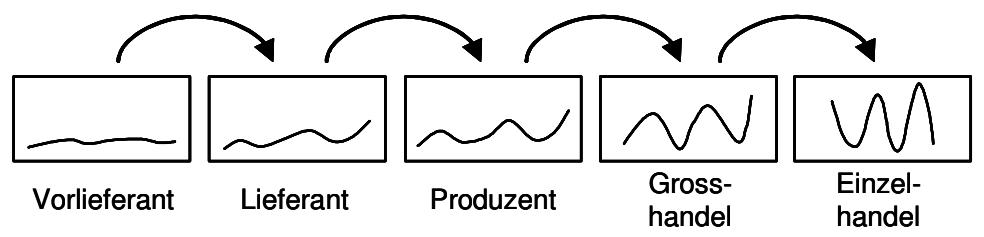

Abbildung 19: Kosten- und Kalkulationsdynamik in Supply Chains.

Sachziele der Qualitätsverbesserung sowie der Realisierung von Zeitvorteilen zur Erhöhung des Endkundennutzens sind nur unter Berücksichtigung der dafür entstehenden Kosten zu erreichen. Insofern gilt es, die Maßnahmen zur Zielerreichung unternehmensübergreifend kostenmäßig zu erfassen. Die durch die Endkunden angestoßenen Veränderungen von Zeit- und Qualitätsaspekten in den einbezogenen Unternehmen der Supply Chain wirken sich auf die gesamte Kostenentstehung aus und haben somit auch einen Einfluss auf Kostenniveau, Kostenstruktur und Kostenverlauf.

Auch die Konfiguration von Wertschöpfungsnetzwerken stellt Anforderungen an das Kostenmanagement. Veränderungen in der Supply Chain-Struktur und den Supply Chain-Prozessen sind wertmäßig abzubilden. Im Zusammenhang mit der Supply ChainStruktur stellt sich z.B. die Frage, wie die Kosten durch die Veränderung der Struktur des Wertschöpfungsnetzwerks positiv beeinflusst werden können. Folgende Beispiele aus dyadischen Beziehungen gelten sinngemäß auch im Supply Chain-Kontext. So lassen sich durch die Reduktion von Lieferanten ein höheres Beschaffungsvolumen je Lieferant und damit geringere Materialkosten realisieren. Mit der Auslagerung von Wertschöpfungsaktivitäten ist eine Variabilisierung von Fixkosten verbunden, die sich positiv auf die Kostenstruktur auswirkt. Auf Basis der Supply Chain-Struktur ergeben sich Anforderungen an die Identifikation und Beeinflussung der Kosten von Geschäftsprozessen. So eröffnet eine Lieferantenentwicklung Potenziale zur Qualitätsverbesserung. Dadurch lassen sich kostenintensiver Eingangskontrollen und Doppelarbeiten reduzieren. Mit der Identifikation von Mehrkosten bei Prozessabweichungen trifft das Kostenmanagement Aussagen, die Entscheidungen über zusätzliche Investitionen mit Blick auf spezifische 
Kosteneinsparungsmöglichkeiten erlauben. Zudem kann auch durch die Einführung von Informations- und Kommunikationssystemen eine Verbesserung der Prozesseffizienz realisiert werden. Letztlich ist die Kostenbeeinflussung von Entscheidungen über Supply Chain-Managementkomponenten von Bedeutung. So ermöglichen innovative unternehmensübergreifende Logistikkonzepte wie z.B. eine bedarfssynchrone Anlieferung (Justin-Time) eine Reduktion von Zwischenlagern und damit eine Verringerung der Lagerhaus- und der gebundenen Kapitalkosten.

Auch die Objekte des Supply Chain Management stellen Anforderungen an die Maßnahmen zur Kostenbeeinflussung. Bezogen auf die Material- und Warenflüsse geht damit die Forderung nach einer wertschöpfungsbezogenen Kostenrechnung einher. Ebenso hat das Kostenmanagement die Informationen, die in einer Supply Chain im Zusammenhang mit dem Material- und Warenfluss anfallen, aufzugreifen und antizipativ in Kostengrößen auszudrücken, um bspw. Auswirkungen prognostizierter Nachfrageschwankungen wertmäßig und unternehmensübergreifend zu erfassen. Diese Planungsansätze verlangen eine unternehmensübergreifend angelegte Verknüpfung der Produktions- und der Absatzplanung mit dem Kostenmanagement.

Das Kostenmanagement in Supply Chains spielt weiterhin bei der Betrachtung von Finanzflüssen eine Rolle. Beschaffungsseitig lässt sich über die Gestaltung der Zahlungsziele (Kreditorenmanagement) eine Reduzierung von Kosten erzielen. So müssen sich z.B. Lieferantenkredite wertmäßig abbilden lassen, um die Auswirkungen der gewährten Kredite auf die Kostenstruktur bzw. den Kostenverlauf bewerten zu können. In der Kalkulation sind dabei durch Lieferanten gewährte, niedrige Einstandspreise zu berücksichtigen. Absatzseitig sind mit Hilfe eines unternehmensübergreifenden Kostenmanagement die die Rechnungsstellungssysteme sowie die Zahlungsverfahren wertmäßig abzubilden. ${ }^{1}$ Neben der Betrachtung der Finanzflüsse in Supply Chains stellen gemeinsam getätigte Investitionen einen weiteren Aspekt des unternehmensübergreifenden Kostenmanagements dar. Als Beispiel sind die Auswirkungen des Erwerbs bzw. Leasings von Supply Chain-spezifischen Investitionsgütern auf die Kostenstruktur im Sinne der Zuordnung von Bereitschafts- bzw. Leistungskosten zu nennen. ${ }^{2}$

Aus dem operativen Management der Supply Chain ergeben sich Anforderungen bezüglich der Messbarkeit und Steuerung in den vorgegebenen Supply Chain-Strukturen. Dafür ist es notwendig, dass Kosteninformationen kurzfristig zur Verfügung stehen. Bei Nachfrageschwankungen müssen Informationen über den Kostenverlauf vorliegen, um bei wachsender Nachfrage überproportional ansteigende Kosten im Wertschöpfungsnetzwerk zu vermeiden. Daneben muss das Kostenmanagement für das strategische Management der Supply Chain Informationen bereit stellen, die in die Planung von Strukturveränderungen oder die Änderung der Sachziele einfließen. Dafür ist erforderlich, dass eine Kostentransparenz sowohl in der Breite (mehrere Aktivitäten auf einer Wert-

$\mathrm{Zu}$ beschaffungsseitigen und distributionsseitigen Wertschöpfungseffekten vgl. Pfohl/Elbert/Hofmann (2003), S. 34ff.

2 Vgl. Steinmüller (2003), S. 171ff.; Feinen (2003), S. 187ff. 
schöpfungsstufe) als auch in der Länge (über mehrere Wertschöpfungsstufen hinweg) des Wertschöpfungsnetzwerks vorhanden ist, um die Aufgaben der Gestaltung (Konfiguration) der Supply Chain erfüllen und Investitionen auf Basis der bestehenden Kostensituation planen, bewerten und umsetzen zu können. Neben der Entscheidung über Lieferantenanzahl, einzubeziehende logistische Dienstleister, Standortwahl für Produktionsund Lagerorte können dies auch Veränderungen der Beschaffungs- oder Distributionsstrukturen sein. ${ }^{1}$

Die aufgeführten Anforderungen verdeutlichen den Bedarf eines unternehmensübergreifenden Kostenmanagement. LaLonde und Pohlen (1996) stellen das Supply Chain Costing als einen prozessorientierten Ansatz dar, mit dem eine wertmäßige Abbildung des Material-, Waren- und Informationsflusses über mehrere Wertschöpfungsstufen hinweg gelingen soll. ${ }^{2}$ Dies ermöglicht die Ermittlung der Gesamteffizienz des Wertschöpfungsnetzwerkes sowie eine Effizienzmessung einzelner Prozesse. Die Messbarkeit der Kosten alleine ist jedoch nicht ausreichend. Vielmehr muss auch die Beeinflussung der Kosten im Supply Chain Costing Beachtung finden. Unter dem Begriff des "Interorganisational Cost Management" fassen Cooper und Slagmulder die Aufgaben des Supply Chain Costing als einen systematischen Ansatz zur Koordination von Unternehmensaktivitäten zur Senkung von Kosten in Wertschöpfungsnetzwerken zusammen. ${ }^{3}$ Dabei unterstreichen sie die Maßnahmen zur Kostensenkung, wohingegen die Beeinflussung von Kostenstruktur, -verlauf und -niveau nachgeordnet thematisiert werden. In einer Ausarbeitung von Seuring (2001) wird das Supply Chain Costing modifiziert, indem eine Differenzierung in die (nicht überschneidungsfreien) Kategorien Einzelkosten, Prozesskosten und wertschöpfungskettenweite Transaktionskosten erfolgt, um die Möglichkeiten der Kostenbeeinflussung besser erkennen und die Kostentransparenz erhöhen zu können. ${ }^{4}$

Zusammengefasst spielt neben der Messbarkeit und der Differenzierung nach Kostenkategorien auch die Kostenbeeinflussung von Elementen des konzeptionellen Bezugsrahmens im Supply Chain Management eine bedeutende Rolle. Nach der hier vertretenen Auffassung ist das Supply Chain Costing somit derzeit mit Blick auf seinen Entwicklungsstand eher als methodisch-instrumentellen Ansatz denn als Bezugsrahmen zu interpretieren. Künftig gilt es, mit Blick auf die vielfältigen Anforderungen an der Konzeption eines Kostenmanagement für Supply Chains und seiner Umsetzung zu arbeiten. ${ }^{5}$ Da Wertschöpfungsnetzwerke in der Regel hinsichtlich ihrer Gestaltung und Steuerung ausgeprägte Spezifika aufweisen, benötigt das Supply Chain Costing ein Set an Methoden sind Instrumenten, die dann jeweils im Hinblick auf die konkret verfolgten Ziele geprüft

\footnotetext{
Vgl. Corsten/Gössinger (2001), S. 99.

Vgl. LaLonde/Pohlen (1996), S. 1ff.

Vgl. Cooper/Slagmulder (1999), S. 1.

Vgl. Seuring (2001), S. 114ff. Dabei werden jeweils völlig eigenständige Systematisierungskategorien nicht unbedingt nachvollziehbar zusammengeführt. Dies beeinträchtigt stark die Aussagekraft der daraus abgeleiteten Erkenntnisse.

5 Vgl. Stölzle/Heusler/Karrer (2001), S. 76.
} 
und eingesetzt werden müssen. Dazu kann eine Anleihe bei bekannten Methoden und Instrumenten des Kostenmanagement genommen werden.

\section{Ausgewählte Instrumente für das Supply Chain Costing}

In Anlehnung an die Gestaltungsobjekte des Kostenmanagement bietet sich bei der Auswahl der Instrumente für das Supply Chain Costing eine Orientierung an Produkten, Prozessen und eingesetzten Produktionsfaktoren an. So findet in Bezug auf das Kostenmanagement mit einer produktbezogenen Betrachtung die lebenszyklusorientierte Kostenrechnung eine genauere Betrachtung, die bereits in frühen Phasen des Produktlebenszyklus angreift und beispielsweise Entwicklungslieferanten mit einbezieht. Die Prozessorientierung der Material-, Waren- und Informationsflüsse in Supply Chains kann mit Hilfe der unternehmensübergreifenden Prozesskostenrechnung aufgegriffen werden. Wie die unternehmensübergreifende Prozesskostenrechnung zielt auch der Ansatz der Total Cost of Ownership (TCO) auf die Kosten unternehmensübergreifender Geschäftsprozesse ab, fokussiert aber stärker die spezifischen Investitionen in Gestalt der Infrastruktur und damit die relevanten Produktionsfaktoren. Es wird jeweils geprüft, inwieweit die Instrumente in der Lage sind, die Anforderungen des Kostenmanagement in Supply Chains zu erfüllen.

\subsection{Lebenszyklusorientierte Kostenrechnung}

Die lebenszyklusorientierte Kostenrechnung ist durch eine produktbezogene Betrachtung charakterisiert. ${ }^{1}$ Dabei ist die Erkenntnis maßgebend, dass der Großteil der Produktkosten bereits in der Phase der Produktentwicklung festgelegt wird, in die häufig eine Vielzahl von Lieferanten und Dienstleistungsunternehmen eingebunden sind. Empirische Untersuchungen haben ergeben, dass in dieser Phase bis zu 90\% der gesamten Produktkosten bestimmt werden. ${ }^{2}$ Ein lediglich auf die Kostenbeeinflussung in der Herstellung fokussiertes Kostenmanagement greift demnach zu kurz. Das Kostenmanagement muss bereits in einer frühen Phase der Produktentwicklung beginnen. Dies bedeutet, die erbrachten Leistungen möglichst aller Supply Chain-Akteure wertmäßig zu erfassen. Dazu gehören Vor- und Nachlaufkosten über den gesamten Produktlebenszyklus, insbesondere Logistik- und sonstige Dienstleistungskosten. Weiterhin gilt es, Substitutionsmöglichkeiten zwischen Vorlaufkosten sowie Herstell- und Nachlaufkosten zu analysieren. Die

Neben Lebenszyklusmodellen für Produkte lassen sich auch andere Bezugs- oder Rechenobjekte wie Potenzialfaktoren und Unternehmen bzw. Organisationen nennen. Vgl. Zehbold (1996), S. 14ff.

Vgl. Berliner/Brimson (1988), S. 139f. 
Notwendigkeit derartiger Analysen zeigt sich besonders bei Produktinnovationszyklen, die mit der zügigen Entwicklung und Realisierung von Nachfolgeprodukten immer kürzer werden und eine besonders koordinierte Zusammenarbeit der Supply Chain-Akteure verlangen. Das Kostenmanagement hat daher nicht nur Produktinnovations-, sondern insbesondere auch Prozessinnovationskosten zu beachten. Aus Sicht der Anbieter erweitert sich der Produktlebenszyklus bspw. durch gesetzliche Verpflichtungen um die Phase der Rücknahme sowie Entsorgung von Produkten und muss ebenfalls in der Produktlebenszykluskostenbetrachtung berücksichtigt werden. Eine Erweiterung könnte die Produktlebenszykluskostenrechnung durch die Berücksichtigung von Geschäftsbeziehungen zwischen den Supply Chain-Akteuren erfahren. Denn diese Geschäftsbeziehungen unterliegen i.d.R. auch einem Lebenszyklus, der sich ebenfalls auf die gesamten Produktkosten auswirkt.

In der Literatur finden sich verschiedene Ausprägungsformen der lebenszyklusorientierten Kostenrechnung. ${ }^{1}$ Kennzeichnend sind eine strategische Orientierung und eine ganzheitliche Betrachtungsweise. Die betrachteten Produkte haben für die Unternehmen eine strategische Bedeutung, weil die mit ihnen verbundenen Erfolgswirkungen einen längerfristigen Zeitraum betreffen. Das Merkmal der Ganzheitlichkeit erfüllt die lebenszyklusorientierte Kostenrechnung, da sie alle mit dem Objekt verbundenen Phasen und Aktivitäten umfasst und sich nicht auf eine Periode des Lebenszyklus beschränkt. Sie betrachtet den gesamten Lebenszyklus eines Produktes als Investitionsprojekt und ist als Verbindung von Kostenrechnung und Investitionsrechnung zu interpretieren. ${ }^{2}$ Mit der Lebenszyklusorientierung wird eine auf den zukünftigen Restlebenszyklus ausgerichtete Planungsrechnung abgebildet, die im weiteren Verlauf aufgrund gewonnener Erfahrungen fortlaufend zu verfeinern ist. Für den bereits abgelaufenen Produktlebenszyklus dagegen fungiert sie als Kontrollrechnung und kann somit das bisherige Kostenniveau überprüfen, Abweichungen im Kostenverlauf und in der Kostenstruktur aufzeigen sowie Gegenmaßnahmen initiieren. ${ }^{3}$

Zu den Aufgaben der lebenszyklusorientierten Kostenrechnung gehört die Berücksichtigung unterschiedlicher Ausprägungen der Markt- und Absatzbedingungen im Laufe des Produktlebenszyklus. Ziel ist es, die Produktlebenszykluskosten gering zu halten bzw. den Produktlebenszyklusgewinn zu erhöhen. Die ganzheitliche Ausrichtung verlangt zudem, langfristig die Produktrentabilität zu messen und die wertmäßigen Konsequenzen sowohl technischer als auch logistischer Alternativen bereits in den frühen Phasen des Produktlebenszyklus aufzuzeigen. ${ }^{4}$

Ein einheitlich akzeptiertes Verfahren der lebenszyklusorientierten Kostenrechnung lässt sich aufgrund diversen Rechenvarianten mit unterschiedlichen methodischem Aufbau in

Erste Anwendungen finden sich in der industriellen Großserienproduktion bzw. für komplexe Produkte wie Industrieanlagen. Vgl. Riezler (2002), S. 208.

Vgl. Back-Hock (1992), S. 703.

Vgl. Baden (1997), S. 81.

Vgl. Back-Hock (1992), S. $703 f$. 
der einschlägigen Literatur nicht identifizieren. Ohne ins Detail gehen zu können, ergeben sich für die lebenszyklusorientierte Kostenrechnung drei grundsätzliche Ausgestaltungsvarianten. ${ }^{1}$ In der ersten Form baut sie auf der Grenzplankosten- und Deckungsbeitragsrechnung auf, die durch eine mehrperiodige Kostenerfassung und -auswertung erweitert wird. $^{2}$ Dabei bedient sie sich der Amortisationsrechnung, mit der dem über den Produktlebenszyklus diskontinuierlichen Anfall von Kosten Rechnung getragen und über den Kapitalwert ein produktbezogenes Entscheidungskriterium ermittelt wird. Mit der periodenübergreifenden Rechnung sind jedoch Verrechnungsprobleme der Gemeinkosten verbunden. ${ }^{3}$ In die zweite Form auf Basis diskontierter Ein- und Auszahlungen findet die dynamische Investitionsrechnung Eingang. Mit der Betrachtung der Produktlebenszyklen werden die anfallenden Zahlungen periodengerecht zugeordnet. ${ }^{4}$ Bei der dritten Ausgestaltung auf Basis der relativen Einzelkosten- und Deckungsbeitragsrechnung finden ausschließlich Aus- und Einzahlungen als Rechengrößen Verwendung. Hier wird auf die Zurechnung periodenbezogener Gemeinkosten verzichtet. Die erforderlichen wert- und mengenmäßigen Grunddaten wie Einzelkosten und Produktmengen sind für den gesamten Produktlebenszyklus zu schätzen und in einer zweckneutralen Grundrechnung für jede Periode zu erfassen. ${ }^{5}$ Die Ausgestaltung der lebenszyklusorientierten Kostenrechnung ist mit Problemen der methodischen Ausgestaltung behaftet. Dies betrifft bspw. die unternehmensübergreifende Abgrenzung verschiedener Produktlebenszyklusphasen. Weitere Schwierigkeiten stellen die unternehmensübergreifend zu harmonisierende Kostenerfassung sowie die Abhängigkeit von Planungsrechnungen und Prognosen über den gesamten Lebenszyklus dar, die bei den beteiligten Supply Chain-Akteuren ebenfalls verschiedene Ausprägungen haben können. Insgesamt steht der Umsetzung der lebenszyklusorientierten Kostenrechnung eine beachtliche Komplexität entgegen.

Ein Vorteil der lebenszyklusorientierten Kostenrechnung kann in der Aufdeckung von Schwachstellen im Innovationsprozess sowie in der expliziten Einbindung von Supply Chain-Akteuren gesehen werden. Bei identifizierten Budgetengpässen besteht die Notwendigkeit, kostenintensiver Aktivitäten innerhalb der Supply Chain abzustimmen. Damit werden unter Einhaltung der Qualitätsanforderungen an den Produkten und den notwendigen Prozessen Lösungen mit geringeren Produktlebenszykluskosten gesucht. Durch die Einbeziehung von Lieferanten und Dienstleistungsunternehmen einerseits sowie dem Kundenverhalten andererseits werden die vom Supply Chain Management geforderten netzwerk- und marktbezogenen Kostendeterminanten berücksichtigt. Schwerpunktmäßig wirkt die lebenszyklusorientierte Kostenrechnung in den frühen Phasen des Produktlebenszyklus. Kosten, die im Marktzyklus nach erfolgter Produkteinführung anfallen, lassen sich mit diesem Instrument nur unzureichend transparent machen. Ein ope-

\footnotetext{
Einen umfangreichen Überblick der Ausgestaltungsvarianten der lebenszyklusorientierten Kostenrechnung findet sich in Baden (1997), S. 84ff.

Vgl. Zehbold (1996), S. 153f.

Vgl. Baden (1997), S. 104.

Vgl. Zehbold (1996), S. 193ff.

Vgl. Back-Hock (1992), S. 703.
} 
ratives Kostenmanagement in Supply Chains ist damit kaum möglich. Zudem stellt die Definition der "relevanten" Kosten ein Problem dar, haben doch verschiedene Akteure in der Supply Chain jeweils eigene Sichtweisen auf die Relevanz einzelner Kostenkategorien. Ausgestaltet als Vollkostenrechnung, werden mittelbar produktbezogene Kosten den Produkten bzw. Produktgruppen mit Hilfe von Kostenschlüsseln zugerechnet. Dies betrifft i.d.R. auch die Logistikkosten, so dass erhebliche Unschärfen in der Zuordnung der im Supply Chain-Kontext relevanten Kostenkategorien entstehen. Ein weiterer Nachteil kann sich durch die mangelnde Akzeptanz des Verfahrens bei Supply ChainAkteuren einerseits und Mitarbeitern in eigenen Unternehmen andererseits entstehen, wenn Daten von Supply Chain-Akteuren quasi extern vorgegeben, aber als nicht plausibel erachtet werden. Die damit reduzierte Nachvollziehbarkeit der Kosteninformationen gefährdet ein zentrales Ziel des Kostenmanagement in Supply Chains, das Schaffen von Kostentransparenz über die Produktkosten in Abhängigkeit des Lebenszyklus. Eine Abhilfe ist in der Verknüpfung der lebenszyklusorientierten Kostenrechnung mit der unternehmensübergreifenden Prozesskostenrechnung zu sehen.

\subsection{Unternehmensübergreifende Prozesskostenrechnung}

Die Prozesskostenrechnung wird in der wissenschaftlichen Literatur umfangreich behandelt. ${ }^{1}$ Im Logistikbereich hat die Prozesskostenrechnung aufgrund ihrer ausgeprägten Querschnittsorientierung bereits früh in die unternehmerische Praxis Eingang gefunden. ${ }^{2}$ Aufgabe der Prozesskostenrechnung ist die Reduzierung von Ungenauigkeiten in der Kostenstruktur indirekter Leistungsbereiche. Mit der Prozesskostenrechnung wird durch eine veränderte Gemeinkostenzurechnung eine verbesserte Transparenz der Kosten betrieblicher Prozesse verfolgt. ${ }^{3}$ Durch eine systematische Erfassung der betroffenen Prozesse, eine Identifikation von Kostentreibern und eine Definition leistungsbezogener Größen für die Prozesse in den indirekten Bereichen können die Gemeinkosten auf definierte Prozessleistungen zugerechnet werden. Somit erfolgt die Verrechnung der Kosten auf Kostenträger nicht nach pauschalen Gemeinkostenzuschlägen, sondern nach den tatsächlich in Anspruch genommenen Prozessleistungen. In der Logistik bewirkt der Umfang an vorbereitenden, planenden, steuernden und überwachenden Tätigkeiten im $\mathrm{Zu}$ sammenhang mit den Material-, Waren- und Informationsflüssen einen vergleichsweise hohen Anteil der Gemeinkosten, so dass besonders hier die Prozesskostenrechnung für zusätzliche Kostentransparenz sorgen kann. ${ }^{4}$

\footnotetext{
Vgl. bspw. Kaplan/Cooper (1999); Horváth/Mayer (1995); Pfohl/Stölzle (1991); Franz (1990); Horváth/Mayer (1989).

Vgl. Weber (2002a), S. 52.

Vgl. Coenenberg (2003), S. 222; Lin/Collins/Su (2001), S. 704.

Vgl. Coenenberg (2003), S. 206f.
} 
Trotz der genannten Vorteile erfährt die Prozesskostenrechnung in der unternehmerischen Praxis bislang nur eine vergleichsweise geringe Verbreitung. ${ }^{1} \mathrm{Zu}$ begründen ist dieser niedrige Umsetzungsgrad durch den recht hohen Aufwand, den die Einführung und Anwendung einer Prozesskostenrechnung von einem Unternehmen verlangt. Darüber hinaus ist in den Unternehmen häufig keine prozessorientierte, sondern eine funktionale Ausrichtung vorzufinden.

Bislang sind demzufolge auch nur wenige Vorschläge einer unternehmensübergreifenden Prozesskostenrechnung bekannt, welche die Anforderungen des Supply Chain Management umfassend berücksichtigen. Einen ersten konkreten Ansatz entwickelten Dekker und van Goor, die eine punktuelle Prozesskostenrechnung über mehrere Supply Chain-Stufen hinweg vorschlagen. ${ }^{2}$ Dies führt unabhängig von den Vorteilen der Prozesskostenrechnung im unternehmensinternen Bereich dazu, dass mit Hilfe der Prozesskostenrechnung eine von allen beteiligten Supply Chain-Akteuren akzeptierte "Sprache" gefunden werden kann. Die gemeinschaftliche Definition von Prozessen, Prozessleistungen, Kostenkategorien und Kostentreibern spielt daher eine bedeutende Rolle im Kostenmanagement von Supply Chains. Mit Blick auf die Implementierung einer solchen unternehmensübergreifenden Prozesskostenrechnung erscheint es sinnvoll, eine stufenweise Einführung vorzunehmen, damit auch solche Akteure, die bisher über keine Erfahrungen mit der Prozesskostenrechnung verfügen, in das Supply Chain Costing eingebunden werden können. Weber schlägt diesbezüglich eine dreistufige Entwicklung der unternehmensübergreifenden Prozesskostenrechnung vor. ${ }^{3}$ In einem ersten Schritt erfolgt eine Effizienzsteigerung über Mengenansätze (bspw. Lagerbestände oder Verfügungsgrade), nicht jedoch über die Berechnung von Kosteneinsparungen (so genannte unternehmensübergreifende Kostenoptimierung mittels Kostentreiber). Dabei muss für die Erreichung des Ziels einer unternehmensübergreifenden Kostenreduktion nicht notwendig eine voll ausgebaute Prozesskostenrechnung etabliert werden. Jedoch sollte ein grundlegendes Verständnis über die zu optimierenden Prozesse und die wesentlichen Kostentreiber geschaffen werden. Anschließend werden den Prozessen in der Supply Chain fallweise Kosten zugeordnet, wobei dies punktuell erfolgen kann (so genannte partielle unternehmensübergreifende Prozesskostenrechnung) und somit auch keine laufende Leistungserfassung erforderlich ist. Mit der zunehmenden Verbreitung ergeben sich dann schrittweise weitere Anwendungsmöglichkeiten einer unternehmensübergreifenden Prozesskostenrechnung, beispielsweise im Hinblick auf eine differenzierte Kalkulation von Prozesskosten für Produkte, Kunden oder Distributionskanälen.

Eine mögliche Strukturierung der Implementierung der unternehmensübergreifenden Prozesskostenrechnung kann über die in Abbildung 20: Implementierungsschritte der unternehmensübergreifenden Prozesskostenrechnung

Vgl. Weber (2002b), S. 212. 40\% der deutschen Unternehmen betreiben die Prozesskostenrechnung fallweise und lediglich $4 \%$ haben die Prozesskostenrechnung im vollen Umfang implementiert. Vgl. Homburg u.a. (1998).

2 Vgl. Dekker/van Goor (2000), S. 41ff.

3 Vgl. Weber (2002b), S. 212ff. 
dargestellten, neun Schritte erfolgen. ${ }^{1}$

\begin{tabular}{|l|}
\hline 1. Identifikation der relevanten Supply Chain-Akteure \\
\hline 2. Einigung über ein gemeinsames Vorgehen (evtl. Vertragsgestaltung) \\
\hline 3. Auswahl und Analyse der relevanten Supply Chain Prozesse \\
\hline 4. Aufspaltung der Prozesse in Aktivitäten \\
\hline 5. Bestimmung der nötigen Produktionsfaktoren \\
\hline 6. Ermittlung und Bewertung der Aktivitätskosten \\
\hline 7. Ermittlung und Einigung auf Prozesskostensätze \\
\hline 8. Analyse und Simulation \\
\hline 9. Abweichungsanalyse \\
\hline
\end{tabular}

Abbildung 20: Implementierungsschritte der unternehmensübergreifenden Prozesskostenrechnung

Quelle: In Anlehnung an Schlüchtermann/Völkl (2004), S. 386).

Das Ziel der ersten Schritte umfasst die Abbildung der Supply Chain in einem einheitlichen Prozessmodell zur Ermittlung der Kosten sowie zur Ermöglichung eines unternehmensübergreifenden Kostenvergleichs. Beginnend mit der Identifikation der relevanten Supply Chain-Akteure und der Einigung auf ein gemeinsames Vorgehen gilt es, die Hauptprozesse der beteiligten Unternehmen unter Zuhilfenahme von Supply Chain Management-Referenzmodellen zu erfassen. ${ }^{2}$ Hauptprozesse sind in der Regel hoch aggregiert und werden deshalb weiter in Teilprozesse und Aktivitäten untergliedert. ${ }^{3}$ Bei den Aktivitäten sollte über den betrachteten Supply Chain-Abschnitt ein einheitliches Aggregationsniveau eingehalten werden, um Aktivitäten gleichen Umfangs der beteiligten Unternehmen gegenüber stellen zu können. Als weitere Differenzierung im Rahmen der

Zu den folgenden Ausführungen vgl. Schlüchtermann/Völkl (2004), S. 386ff. sowie in ähnlicher Form auch Lin/Collins/Su (2001), S. 706ff.; LaLonde/Pohlen (1996), S. 5ff.

2 Mit dem Supply Chain Operations Reference-Modell (SCOR) wurde ein einheitliches und vergleichbares Prozessmodell für Supply Chains entwickelt, in dem die grundlegende Gestaltung von Strukturen und Prozessen der gesamten Supply Chain unterstützt wird. Auf weitere Referenzmodelle soll an dieser Stelle nicht weiter eingegangen werden. Für einen umfangreichen Überblick siehe bspw. Heusler (2004), S. 79ff.

3 Unter einer Aktivität versteht man die Abbildung operativer Tätigkeiten einer Kostenstelle. Bspw. könnten für die Logistikkostenstelle "Warenannahme" die Aktivitäten "Paletten entladen mit Gabelstapler", "Manuelle Warenerfassung" und "Stichprobenweise Kontrolle" definiert werden. Vgl. Reichmann (2001), S. $168 f$. 
Analyse erfolgt die Bestimmung der dazu benötigten Produktionsfaktoren. Jeder mit Kosten bewertete Verbrauch von Produktionsfaktoren wird im nächsten Schritt anteilig auf die Aktivitäten zugeteilt. Die Aktivitätskosten umfassen somit die Summe aller bewerteten Produktionsfaktorverbräuche und sind mit der zugehörigen Leistung ins Verhältnis zu setzen, um daraus den jeweiligen Aktivitätskostensatz zu ermitteln. Eine schrittweise Zusammenfassung mehrerer Aktivitäten zu einem Teil- und einem Hauptprozess erlaubt die Ableitung von Prozesskostensätzen. Mit der Ermittlung der Prozesskostensätze für gleichartige Prozesse können anschließend die beteiligten Unternehmen verglichen werden, um daraus Effizienzaussagen für die Prozessdurchführung abzuleiten. Danach besteht die Möglichkeit, die Kostentreiber der Prozesse in der Supply Chain zu ermitteln und alternative Supply Chain-Konfigurationen zu bewerten. Dabei zeigt sich, dass der Erfolg einer Änderung nicht alleine von Prozesskostensätzen, sondern auch von den damit erzielten Veränderungen in den Prozessleistungen abhängt und somit sowohl wert- als auch leistungsbezogene Kriterien zu berücksichtigen sind. Ferner lassen sich im Rahmen einer Abweichungsanalyse Kostensenkungspotenziale evaluieren, wobei neben wert- auch leistungsbezogene Abweichungen zu ermitteln sind. Abschliessend wird in einer Feedback-Schleife hinterfragt, ob die unternehmensübergreifende Prozesskostenrechnung auf weitere Supply Chain-Akteure ausgedehnt oder aufgrund einer hohen Komplexität auf wenige Akteure beschränkt werden soll. Mit jeder Veränderung der einbezogenen Supply Chain-Akteure sind die Implementierungsschritte erneut zu durchlaufen.

Traditionelle Kostenrechnungssysteme beziehen sich im Schwerpunkt auf direkte Leistungsbereiche, wobei kostenstellenübergreifende Aktivitäten insbesondere in produktfernen Bereichen keine adäquate Berücksichtigung finden. Die Prozesskostenrechnung deckt die kostenstellenübergreifenden Aktivitäten dagegen ab. Ein weiterer Vorteil der Prozesskostenrechnung ist in der Unterstützung der lebenszyklusorientierten Kostenrechnung zu sehen. Als vollkostenorientiertes Instrument umfasst die lebenszyklusorientierte Kostenrechnung nicht nur die Einzel-, sondern auch die Gemeinkosten. Durch den Einsatz der Prozesskostenrechnung für die Gemeinkosten der lebenszyklusorientierten Rechnung lassen sich die Gemeinkosten verursachungsgerecht den Prozessen im Produktlebenszyklus zurechnen.

Im unternehmensübergreifenden Kontext ist die Prozesskostenrechnung mit einigen besonderen Problemen konfrontiert. So lässt sich eine Harmonisierung der Prozessdefinition bei den beteiligten Supply Chain-Akteuren schwer durchsetzen, wenn bereits eine unternehmensbezogene Prozesskostenrechnung besteht. Ebenso ist die einheitliche Zurechnung von Prozesskosten auf Teil- und Hauptprozesse nicht leicht zu realisieren. So kann ein Prozess für den einen Supply Chain-Akteur einen Hauptprozess, für den anderen einen Teilprozess darstellen. Mit diesen Abgrenzungsproblemen geht auch oft eine unterschiedliche Identifikation von Kostenkategorien und Kostentreibern einher, woraus Barrieren für eine entsprechende Harmonisierung für den betrachteten Supply ChainAusschnitt entstehen. 
Die Prozesskostenrechnung stellt ein Instrument dar, das einige der in Abschnitt 2.4 genannten Anforderungen an ein Supply Chain Costing erfüllt. Über die verbesserte Prozesstransparenz lassen sich Kosteneinsparungspotenziale realisieren, da anhand der Gegenüberstellung von Prozessschritten Redundanzen identifiziert werden können. Mit standardisierten Kostenkategorien und einheitlichen Kostentreibern wird eine in der Supply Chain abgestimmte Entscheidungsfindung unterstützt, welcher Supply ChainAkteur welche Prozesse zu welchen Kosten realisieren kann. Daneben erlaubt die erhöhte Kostentransparenz einen Supply Chain-weiten Kostenvergleich und ermöglicht die Identifikation von gemeinsamen Kostensenkungspotenzialen. Schliesslich lassen sich Veränderungen in der Supply Chain-Struktur und den Geschäftsprozessen kostenmässig abbilden. Bei einer ausreichend weiten Fokussierung nähert sich die Prozesskostenrechnung damit einer Total Cost of Ownership-Betrachtung an.

\subsection{Total Cost of Ownership}

Der Ansatz der Total Cost of Ownership (TCO) zielt primär auf die Kosten ab, die aufgrund der Verfügung über Produktionsfaktoren anfallen. Angesprochen werden dabei sowohl direkte (budgetierbare) als auch indirekte (Opportunitäts-)Kosten. Direkte Kosten sind in diesem Zusammenhang Kosten, welche mit der Beschaffung, der Bereitstellung und dem Betrieb einer Infrastruktur bzw. von Produktionsfaktoren entstehen. Opportunitätskosten resultieren demgegenüber aus Produktivitätsverlusten, die dadurch entstehen, dass der "Owner" Ressourcen für die Aufrechterhaltung von Infrastruktur bzw. Produktionsfaktoren bereitstellen muss (z.B. Schulungen oder Wartung) und damit diese Ressourcen anderen Verwendungen (z.B. Leistungsprozessen) entzogen werden. ${ }^{1}$ Das ursprünglich aus dem Beschaffungs- und Logistikbereich abgeleitete Instrument erhebt den Anspruch, Kostenkategorien, die im Zusammenhang mit dem Material-, Waren- und Informationsfluss bis hin zum Endverbraucher entstehen, zu erfassen. ${ }^{2}$ Dazu gehören alle Kosten, die im Zusammenhang mit der Beschaffung, der Herstellung und der Erhaltung eines Produkts zusammenhängen. Im Hinblick auf eine bilaterale Beziehung konzentriert sich die Betrachtung auf diejenigen Kostenkategorien, die von beiden Akteuren mittelbar und unmittelbar beeinflussbar werden wie z.B. vorgehaltene Lagerkapazitäten.

Bislang hat sich jedoch weder für den Begriff noch für die Einführung und die Anwendung von TCO ein Standard herausgebildet. Dennoch lassen sich beim TCO-Ansatz verschiedene Ausprägungsformen identifizieren. In einer prozessualen Sicht werden die Kostenkategorien nach Maßgabe der Wertschöpfungsstufen differenziert. Damit kann prinzipiell eine Anwendbarkeit im Supply Chain Management herbeigeführt werden. Bei komplexen Netzwerkstrukturen können mehrere Kostenkategorien an den Prozessstufen

Vgl. Treber/Teipel/Schwickert (2004), S. 8.

Vgl. Ellram (1995), S. 4. 
einer Supply Chain ausgerichtet und zu Schlüsselkategorien verdichtet werden. ${ }^{1}$ Die Besonderheit dieses Modells liegt in der schwerpunktmäßigen Identifikation von unternehmensübergreifenden Kostenkategorien, welche sich auf die Infrastruktur und Produktionsfaktoren einzelner Supply Chain-Prozessabschnitte beziehen. Bewertungsfragen sowie die Modalitäten der Erfassung und Verrechnung der einzelnen Kostenkategorien durch die Supply Chain-Akteure werden bei diesem Ansatz allerdings nicht thematisiert.

Eine alternative Auffassung des TCO nimmt Bezug auf das Beschaffungsmanagement, die unterschiedliche Entscheidungsbereiche und die davon betroffenen Kostenkategorien. ${ }^{2}$ Ellram und Siferd differenzieren sechs Kostenkategorien, die das Management, die Auslieferung, den Service, die Kommunikation, die Qualitätssicherung und die Preisgestaltung betreffen. Dabei bieten sie eine umfangreiche Auflistung von Einflussgrößen an, die bei der Ermittlung der TCO zu berücksichtigen sind. Neben dem Hinweis auf die prozessorientierte Abbildung der Kostenkategorien werden jedoch keine konkreten Anhaltspunkte zur Erfassung und Verrechnung der TCO gegeben. Darüber hinaus stellt Ellram mit einer stärkeren Einengung der Betrachtungsweise eine transaktionsorientierte Variante dar. Als Bezugspunkt zur Differenzierung von Kostenkategorien wird die einzelne Transaktion herangezogen, der neben dem Einkaufspreis die Kostenkomponenten der Transaktion selbst sowie die der Transaktion vor- und nachgelagerten Kostenkomponenten zugeordnet werden. ${ }^{3}$ Hierzu zählen die Kosten der Auftragsabwicklung, der Anlieferung, der Verzollung, der Zahlungsmodalitäten sowie nötiger Inspektionsmaßnahmen bis hin zur Nachverfolgung der Transaktion einschließlich notwendiger Korrekturmaßnahmen. Vorgelagerte Komponenten der Transaktion wie die Lieferantenauswahl und -entwicklung sowie nachgelagerte Komponenten wie bspw. Reparaturmaßnahmen werden als eigenständige Kostenkategorien abgebildet. Mit der Unterteilung der Kostenkategorien und der Benennung einzelner Implementierungsschritte kommt der transaktionsorientierten Ausgestaltung der TCO eine vergleichsweise große Umsetzungsnähe zu. ${ }^{4}$ Es ist zu konstatieren, dass die wesentliche Gestaltungskraft der TCO in der Beschaffung sowie im Investitionsbereich zu sehen ist. Aber auch im Supply Chain-Kontext unterstützt die gemeinsame Anwendung des Instruments die Auswahl von Supply ChainAkteuren sowie das Management der Geschäftsbeziehungen.

$\mathrm{Zu}$ den Vorteilen des TCO-Ansatzes zählt die Unterstützung von Entscheidungen im Hinblick auf die Gestaltung der Geschäftsbeziehungen zwischen den Supply ChainAkteuren durch eine erhöhte Kostentransparenz bzgl. der diesen Beziehungen zugrunde liegenden Transaktionen. Mit der gemeinsamen Analyse der Kostenstrukturen geht auch eine intensive Kommunikation zwischen den Akteuren einher. Dies unterstützt den Aufbau eines dauerhafter Beziehungsgefüges, in dem die beteiligten Akteure gemeinsam und kontinuierlich an der Verbesserung der Leistungsprofile und der Identifikation von

Vgl. Cavinato (1992), S. 292ff.

Vgl. Ellram/Siferd (1993), S. 164ff.

Vgl. Ellram (1993), S. 7ff.

Vgl. Stölzle (1999), S. 301. 
Kosteneinsparungspotenzialen arbeiten. ${ }^{1}$ Dies wirkt sich positiv auf die Planungsqualität der Supply Chain-Prozesse aus, etwa in Hinblick auf deren Fehleranfälligkeit oder deren Handhabbarkeit. Die TCO-Betrachtung kann zudem als Anhaltspunkt für ein Supply Chain-weites Benchmarking dienen, um ein erfolgreiches unternehmensübergreifendes Kostenmanagement zu implementieren.

Als Defizite des TCO-Ansatzes sind Fragen der Abbildungsfähigkeit der einzelnen Kostenkategorien zu nennen. Wenn mehrere Supply Chain-Akteure das Instrument gemeinsam einsetzen, ergibt sich ein Abstimmungsbedarf bzgl. der Transaktionsabgrenzung, der Zurechnung einzelner Kostenkategorien auf Transaktionen sowie der Bewertung der transaktionsbezogenen Kosten. Dies bezieht sich auf tolerierbare Ungenauigkeiten ebenso wie auf Änderungen der Rahmenbedingungen und Annahmen bei der Ermittlung transaktionsbezogener Kosten. In Bezug auf das rechentechnische Vorgehen weist die transaktionsorientierte Ausgestaltung mit der Zuordnung von Prozessen, Kostenkomponenten und Kostentreibern das strengste Profil im TCO-Spektrum auf. Dabei ist aufgrund des immanenten Prozessbezugs eine Kombination mit der Prozesskostenrechnung denkbar. Eine weitere Schwäche liegt in der engen Fokussierung auf Kosten. Die Vernachlässigung von Nutzenkomponenten führt zu der Gefahr, dass Effizienzbestrebungen nicht umfassend wahrgenommen werden. Beispielsweise können Produktionsfaktoren mit eigenen Absatzpotenzialen u.U. Rationalisierungsmaßnahmen zum Opfer fallen. Schließlich ist der TCO-Ansatz statisch ausgelegt.. ${ }^{2}$ Hier vermag eine Dynamisierung im Sinne der lebenszyklusorientierten Kostenrechnung Abhilfe schaffen.

\section{Zusammenfassung und Ausblick}

Bislang gibt es im deutschen und englischen Sprachraum nur wenige wissenschaftliche Publikationen, die sich ausführlich mit dem Supply Chain Costing befassen. Im vorliegenden Beitrag werden deshalb ausgehend von der Entwicklung der Logistik zum Supply Chain Management sowie der Entwicklung von der Kostenrechnung zum Kostenmanagement systematisch - unter expliziter Berücksichtigung der Logistikkostenrechnung - die konzeptionellen Grundlagen des Supply Chain Costing vorgestellt.

Es zeigt sich, dass das Supply Chain Management als kooperationsorientierter und unternehmensübergreifender Managementansatz eine vielfältige Ausgestaltung erfährt. Eine Konzeptualisierung des Kostenmanagement ist bislang im Hinblick auf das Supply Chain Management noch nicht umfassend erarbeitet worden. So verwundert es nicht, dass ein konzeptioneller Bezugsrahmen, der das unternehmensübergreifende Kostenmanagement in Supply Chains aufgreift, bislang noch aussteht. Insofern konzentrieren sich

Eine Übersicht über die Nutzenkategorien der TCO findet sich bei Ellram (1993), S. 4. Vgl. auch Ell$\mathrm{ram} /$ Siferd (1993), S. 168ff.

Vgl. Stölzle (1999), S. 303. 
die Verfasser auf den methodisch-instrumentellen Bereich des Supply Chain Costing, der mit dem kombinierten Einsatz mehrerer Instrumente die aufgestellten Anforderungen aus der Übertragung des Kostenmanagement auf Supply Chains zumindest teilweise erfüllt. Einer direkten Übertragung bekannter Kostenmanagementinstrumente auf das Supply Chain Management sind schon aufgrund dessen unternehmensübergreifenden Ausrichtung Grenzen gesetzt. Hier ist eine Anpassung des Kostenmanagement an den Anspruch von Wertschöpfungsnetzwerken notwendig. Insbesondere die aufgezeigten Überschneidungen beim Einsatz von Kostenmanagementinstrumenten bestärken die Forderung nach einer methodisch-instrumentellen Weiterentwicklung.

Forschungsbedarf zum Supply Chain Costing lässt sich im Hinblick auf die Erfassung standardisierter Leistungs- und Kostendaten sowie auf ein unternehmensübergreifendes Prozessverständnis in der Supply Chain konstatieren. Nur nach einem Schließen dieser Lücken erscheint es möglich, die Supply Chain-Kosten zu beeinflussen und die Gesamteffizienz eines Supply Chain-Ausschnitts zu beurteilen. Entscheidungen, die sich auf die Supply Chain-Akteure auswirken (z.B. Maßnahmen zur Komplexitätsreduktion, zur Verringerung von Beständen oder zur Verkürzung der Durchlaufzeiten), könnten sich so auf relevante Kosteninformationen stützen. Ferner wäre dann ein Kosten-Benchmarking zwischen des Supply Chain-Akteuren umsetzbar.

In der näheren Zukunft wird Supply Chain Costing jedoch sicherlich nur vereinzelt zwischen einer jeweils beschränkten Zahl an Supply Chain-Akteuren vorzufinden sein. Die Ausdehnung über ein Wertschöpfungsnetzwerk hinweg kann vermutlich erst dann erfolgen, wenn auch das Konzept des Supply Chain Management an sich verstärkt in die unternehmerische Praxis Eingang findet.

\section{Literaturverzeichnis}

Aberle, G. (2003): Transportwirtschaft: Einzelwirtschaftliche und gesamtwirtschaftliche Grundlagen. 4., überarb. u. erw. Auflage. München / Wien 2003.

Back-Hock, A. (1992): Produktlebenszyklusorientierte Ergebnisrechnung. In: Männel, W. (Hrsg.): Handbuch Kostenrechnung. Wiesbaden 1992, S. 703-714.

Baden, A. (1997): Strategische Kostenrechnung. Einsatzmöglichkeiten und Grenzen. Wiesbaden 1997.

Bechtel, C. / Jayaram, J. (1997): Supply Chain Management. In: The International Journal of Logistics Management 8(1997)1, S. 15-34.

Berliner, C. / Brimson, J.A. (1988): Cost Management for Today`s Advanced Manufacturing. The CAM -I Conceptual Design. Boston 1988.

Bloech, J. (1997): Logistikgeschichte. In: Bloech, J. / Ihde, G.B. (Hrsg.): Vahlens Großes Logistiklexikon. München 1997, S. 577-579. 
Bowersox, D.J. / Closs, D.J. (1996): Logistical Management - The Integrated Supply Chain Process. New York 1996.

Brede, H. (1993): Entwicklungstrends in Kostenrechnung und Kostenmanagement. In: Die Unternehmung 47(1993)4, S. 333-357.

Cavinato, J.L. (1992): A Total Cost / Value Model for Supply Chain Competitiveness. In: Journal of Business Logistics 13(1992)2, S. 285-301.

Christopher, M. / Ryals, L. (1999): Supply Chain Strategy: Its Impact on Shareholder Value. In: The International Journal of Logistics Management 10(1999)1, S. 1-10.

Coenenberg, A.G. (2003): Kostenrechnung und Kostenanalyse. 5. Auflage. Stuttgart 2003.

Cooper, C.C. / Ellram, L.M. (1993): Charateristics of Supply Chain Management and the Implications for Purchasing and Logistics Strategy. In: The International Journal of Logistics Management 4(1993)2, S. 13-24.

Cooper, R. (1988): The Rise of Activity-Based Costing - Part One: What is an AcitivityBased Cost System? In: Journal of Cost Management, Summer(1988), S. 45-54.

Cooper, R. / Slagmulder, R. (1999): Supply Chain Development for the Lean Enterprise - Interorganisational Cost Management. Portland 1999.

Corsten, H. (2001): Ansatzpunkte für ein integratives Kostenmanagement. Schriften zum Produktionsmanagement Nr. 43. Universität Kaiserslautern 2001.

Corsten, H. / Gössinger, R. (2001): Einführung in der Supply Chain Management. München / Wien 2001.

Dekker, H.C. / van Goor, A.R. (2000): Supply Chain Management and Management Accounting: A Case Study of Activity-Based Costing. In: International Journal of Logistics: Research and Applications 3(2000)1, S. 41-52.

Delfmann, W. (1999): Kernelemente der Logistik-Konzeption. In: Pfohl, H.-Chr. (Hrsg.): Logistikforschung. Entwicklungszüge und Gestaltungsansätze. Berlin 1999, S. 37-59.

Dellmann, K. / Franz, K.-P. (1994): Von der Kostenrechnung zum Kostenmanagement. In: Dellmann, K. / Franz, K.-P. (Hrsg.): Neuere Entwicklungen im Kostenmanagement. Bern u.a. 1994, S. 15-30.

Ellram, L.M. (1993): Total Cost of Ownership. Elements and Implementation. In: International Journal of Purchasing and Materials Management 29(1993)4, S. 3-11.

Ellram, L.M. (1995): Total Cost of Ownership. An analysis approach for purchasing. In: International Journal of Physical Distribution \& Logistics Management 25(1995)8, S. 423. 
Ellram, L.M. / Siferd, S.P. (1993): Purchasing. The Cornerstone of the Total Cost of Ownership Concept. In: Journal of Business Logistics 14(1993)1, S. 163-184.

Feinen, K. (2003): Leasing von Logistik-Immobilien. In: Bundesvereinigung Logistik (Hrsg.): Finanzierung - eine neue Dimension der Logistik. Berlin 2003, S. 187-199.

Fischer, Th.M. (2002): Die Kostenrechnung als Basis für das Kostenmanagement. In: Franz, K.-P. / Kajüter, P. (Hrsg.): Kostenmanagement. Wertsteigerung durch systematische Kostensteuerung. Stuttgart 2002, S. 47-58.

Forrester, J. W: (1958): Industrial Dynamics - A Major Breakthrough for Decision Makers. In: Harvard Business Review 36(1958)4, S. 37-66.

Franz, K.-P. (1990): Die Prozesskostenrechnung - Darstellung und Vergleich mit der Plan- und Deckungsbeitragsrechnung. In: Ahlert, D. / Franz, K.-P. / Göppl, H. (Hrsg.): Finanz- und Rechnungswesen als Führungsinstrument. Wiesbaden 1990, S. 109-136.

Franz, K.-P. / Kajüter, P. (1997): Proaktives Kostenmanagement als Daueraufgabe. In: Franz, K.-P. / Kajüter, P. (Hrsg.): Kostenmanagement. Wertsteigerung durch systematische Kostensteuerung. Stuttgart 1997, S. 5-27.

Franz, K.-P. / Kajüter, P. (2000): Kostenmanagement. In: Busse von Colbe, W. / Coenenberg, A.G. / Kajüter, P. / Linnhoff, U. (Hrsg.): Betriebswirtschaft für Führungskräfte. Stuttgart 2000, S. 103-138.

Franz, K.-P. / Kajüter, P. (2002): Proaktives Kostenmanagement. In: Franz, K.-P. / Kajüter, P. (Hrsg.): Kostenmanagement. Wertsteigerung durch systematische Kostensteuerung. Stuttgart 2002, S. 3-32.

Freidank, C.C. (1997): Kostenrechnung. München 1997.

Goldbach, M. (2002): Organizational Settings in Supply Chain Costing. In: Seuring, S. / Goldbach, M. (Hrsg.): Cost Management in Supply Chains. Heidelberg/New York 2002, S. 89-110.

Göpfert, I. (1999): Stand und Entwicklung der Logistik. In: Logistik Management 1(1999)1, S. 19-33.

Göpfert, I. (2002): Einführung, Abgrenzung und Weiterentwicklung des Supply Chain Managements. In: Busch, A. / Dangelmaier, W. (2002): Integriertes Supply Chain Management. Theorie und Praxis effektiver unternehmensübergreifender Geschäftsprozesse. Wiesbaden 2002, S. 25-44.

Heusler, K.F. (2004): Implementierung von Supply Chain Management. Kompetenzorientierte Analyse aus der Perspektive eines Netzwerkakteurs. Wiesbaden 2004.

Hofmann, E. / Elbert, R. (2004): Collaborative Cash Flow Management. Financial Supply Chain Management als Herausforderung der Netzkompetenz. In: Pfohl, H.-Chr. (Hrsg.): Netzkompetenz in Supply Chains. Wiesbaden 2004, S. 93-117. 
Homburg, Chr. / Weber, J. / Aust, R. / Karlshaus, J.T. (1998): Interne Kundenorientierung der Kostenrechnung - Ergebnisse der Koblenzer Studie. Vallendar 1998.

Horváth, P. / Mayer, R. (1989): Prozesskostenrechnung. Der neue Weg zu mehr Kostentransparenz und wirkungsvolleren Unternehmensstrategien. In: Controlling 1(1989)4, S. 214-219.

Horváth, P. / Mayer, R. (1995): Konzeption und Entwicklung der Prozesskostenrechnung. In: Männel, W. (Hrsg.): Prozesskostenrechnung - Bedeutung, Methoden, Branchenerfahrungen, Softwarelösungen. Stuttgart, S. 59-86.

Hummel, W. / Männel, Th. (1986): Kostenrechnung 1: Grundlagen, Aufbau und Anwendungen. 4. Auflage. Wiesbaden 1986.

Ihde, G.B. (2001): Transport, Verkehr, Logistik. Gesamtwirtschaftliche Aspekte und einzelwirtschaftliche Handhabung. 3., völlig überarb. u. erw. Auflage. München 2001.

Joos-Sachse, Th. (2002): Controlling, Kostenrechnung und Kostenmanagement. Grundlagen - Instrumente - Neue Ansätze. 2., überarb. Auflage. Wiesbaden 2002.

Jórasz, W. (1996): Kosten- und Leistungsrechnung. Einführung mit Aufgaben und Lösungen. Stuttgart 1996.

Kajüter, P. (2000): Proaktives Kostenmanagement. Konzeption und Realprofile. Wiesbaden 2000 .

Kajüter, P. (2005): Kostenmanagement in der deutschen Unternehmenspraxis. In: ZfbF 57(2005)2, S. 79-100.

Kaplan, R.S. / Norton, D.P. (1997): Balanced Scorecard: Strategien erfolgreich umsetzen. Stuttgart 1997.

Kaplan, R.S. / Cooper, R. (1999): Prozesskostenrechnung als Managementinstrument. Frankfurt 1999.

Klaus, P. (1999): Logistik als "Weltsicht". In: Weber, J. / Baumgarten, H. (Hrsg.): Handbuch Logistik: Management von Material- und Warenflussprozessen. Stuttgart 1999, S. 15-32.

Kotzab, H. (2000): Zum Wesen von Supply Chain Management vor dem Hintergrund der betriebswirtschaftlichen Logistikkonzeption - erweiterte Überlegungen. In: Wildemann, H. (Hrsg.): Supply Chain Management. München 2000, S. 21-47.

Krüger, R. / Steven, M. (2000): Supply Chain Management im Spannungsfeld von Logistik und Management. In: WiSt 29(2000)9, S. 501-507.

LaLonde, B.J. / Pohlen, T.L. (1996): Issues in Supply Chain Costing. In: The International Journal of Logistics Management 7(1996)1, S. 1-12.

Lambert, D.M. / Cooper, M.C. (2000): Issues in Supply Chain Management. In: Industrial Marketing Management 29(2000)1, S. 65-83. 
Lin, B. / Collin, J. / Su, R.K. (2001): Supply chain costing: an activity-based perspective. In: International Journal of Physical Distribution \& Logistics Management 31(2001)10, S. 702-713.

Männel, W. (1993): Kostenmanagement als Aufgabe der Unternehmensführung. In: krp 37(1993)4, S. 210-213.

Mentzer, J.T. u.a. (2001): Defining supply chain management. In: Journal of Business Logistics 22(2001)2, S. 1-25.

Min, H. / Mentzer, J. (1998): Defining Supply Chain Management within Marketing Strategy. The University of Tennessee 1998.

Otto, A. / Kotzab, H. (2001): Der Beitrag des Supply Chain Managements zum Management von Supply Chains - Überlegungen zu einer unpopulären Frage. In: Schmalenbach ZfbF 53(2001)2, S. 157-176.

Pfohl, H.-Chr. (2004a): Logistiksysteme. Betriebswirtschaftliche Grundlagen. 7., korrig. u. aktual. Auflage. Berlin u.a. 2004.

Pfohl, H.-Chr. (2004b): Logistikmanagement. Konzeption und Funktionen. 2., überarb. u. aktual. Auflage. Berlin u.a. 2004.

Pfohl, H.-Chr. / Elbert, R. / Hofmann, E. (2003): Management der "finanziellen" Supply Chain: Charakterisierung - Aufgabenbereiche - Interdependenzen. In: Bundesvereinigung Logistik (Hrsg.): Finanzierung - eine neue Dimension der Logistik. Berlin 2003, S. $1-64$.

Pfohl, H.-Chr. / Stölzle, W. (1991): Anwendungsbedingungen, Verfahren und Beurteilung der Prozesskostenrechnung in industriellen Unternehmen. In: ZfB 61(1991)11, S. 1281-1305.

Porter, M.E. (1999): Wettbewerbsvorteile: Spitzenleistungen erreichen und behaupten. 3. Auflage. Frankfurt / New York 1999.

Reichmann, Th. (2001): Controlling mit Kennzahlen und Managementberichten. 6., überarb. u. erw. Auflage. München 2001.

Reiß, M. / Corsten, H. (1990): Grundlagen des betriebswirtschaftlichen Kostenmanagements. In: WiSt 19(1990)8, S. 390-396.

Riezler, S. (2002): Produktlebenszykluskostenmanagement. In: Franz, K.-P. / Kajüter, P. (Hrsg.): Kostenmanagement. Wertsteigerung durch systematische Kostensteuerung. Stuttgart 2002, S. 207-223.

Schlüchtermann, J. / Völkl, S. (2004): Rekonfiguration der Logistikaktivitäten in einer Supply Chain mit Hilfe der Prozesskostenrechnung. In: Controlling (2004)7, S. 385-392.

Schötter, J. / Spulak, R. (1989): Techniken des betrieblichen Rechnungswesens. 5. Auflage. München / Wien 1989. 
Schweitzer, M. / Küpper, H.-U. (2003): Systeme der Kosten- und Erlösrechnung. 8. Auflage. München 2003.

Seuring, S. (2001): Supply Chain Costing: Kostenmanagement in der Wertschöpfungskette mit Target Costing und Prozesskostenrechnung. München 2001.

Steinmüller, T. (2003): Finanzierung von Logistikimmobilien. In: Bundesvereinigung Logistik (Hrsg.): Finanzierung - eine neue Dimension der Logistik. Berlin 2003, S. 171185.

Stock, J.R. / Lambert, D.M. (1993): Strategic Logistics Management. 3. Auflage. Homewood/Ill. 1987.

Stölzle, W. (1999): Industrial Relationships. München / Wien 1999.

Stölzle, W. (2002a): Supply Chain Controlling und Performance ManagementKonzeptionelle Herausforderungen für das Supply Chain Management. In: Logistik Management 4(2002)3, S. 10-21.

Stölzle, W. (2002b): Supply Chain Controlling - eine Plattform für die Controlling- und die Logistikforschung? In: Weber, J. / Hirsch, B. (Hrsg.): Controlling als akademische Disziplin. Eine Bestandsaufnahme. Wiesbaden 2002, S. 283-309.

Stölzle, W. / Heusler, K.F. / Karrer, M. (2001): Die Integration der Balanced Scorecard in das Supply Chain Management-Konzept. In: Logistik Management 3(2001)2/3, S. 7385.

Stölzle, W. / Karrer, M. (2004): Von der Unternehmens- zur Supply Chain Performance - ein konzeptioneller Beitrag für das Management von Supply Chains. In: Spengler, T. I Voss, S. / Kopfer, H. (Hrsg.): Logistik Management - Prozesse, Systeme, Ausbildung. Heidelberg 2004, S. 235-254.

Sydow, J. (2002): Zum Management von Logistiknetzwerken. In: Logistik Management 4(2002)2, S. 9-15.

Treber, U. / Teipel, P. / Schwickert, A.C. (2004): Total Cost of Ownership - Stand und Entwicklungstendenzen 2003. Arbeitspapier WI, Nr. 1/2004. Justus-Liebig-Universität Gießen 2004.

Weber, J. (1992): Logistik als Koordinationsfunktion. Zur theoretischen Fundierung der Logistik. In: ZfB 62(1992)8, S. 877-892.

Weber, J. (1997): Einführung in der Rechnungswesen II: Kostenrechnung. 5. Auflage. Stuttgart 1997.

Weber, J. (1999): Ursprünge, praktische Entwicklung und theoretische Einordnung der Logistik. In: Weber, J. / Baumgarten, H. (Hrsg.): Handbuch Logistik: Management von Material- und Warenflussprozessen. Stuttgart 1999, S. 3-14. 
Weber, J. (2002a): Logistikkostenrechnung. Kosten-, Leistungs- und Erlösinformationen zur erfolgsorientierten Steuerung der Logistik. 2., gänzl. überarb. u. erw. Auflage. Berlin u.a. 2002.

Weber, J. (2002b): Logistik- und Supply Chain Controlling. 5., aktual. u. völlig überarb. Auflage. Stuttgart 2002.

Weber, J. / Bacher, A. / Groll, M. (2002): Supply Chain Controlling. In: Busch, A. / Dangelmaier, W. (Hrsg.): Integriertes Supply Chain Management. Theorie und Praxis effektiver unternehmensübergreifender Geschäftsprozesse. Wiesbaden 2002, S. 145-166.

Zehbold, C. (1996): Lebenszykluskostenrechnung. Wiesbaden 1996.

Zöllner, W.A. (1990): Strategische Absatzmarktplanung. Kunden- und Wettbewerbsanalyse für Logistikunternehmen. Berlin u.a. 1990. 